\title{
Influence of structural factors on the tribological performance of organic friction modifiers
}

\author{
Febin CYRIAC*, Xin Yi TEE, Sendhil K. POORNACHARY, Pui Shan CHOW \\ Institute of Chemical and Engineering Sciences, Agency for Science, Technology and Research, Singapore 627833, Singapore \\ Received: 26 November 2019 / Revised: 17 February 2020 / Accepted: 15 March 2020 \\ (C) The author(s) 2020.
}

\begin{abstract}
The influence of structural factors on the lubrication performance of organic friction modifiers (OFMs) formulated in Group V (polyol ester oil) base oil was studied using a ball-on-disk tribometer. The results show that OFMs can mitigate friction under heavy loads, low sliding speeds, and high temperatures. These conditions are commonly encountered in internal-combustion engines between cylinder liners and piston rings. The reduction in friction is ascribed to the boundary lubrication film containing the OFM. The chemical composition analysis of the metal disk surface using energy dispersive X-ray spectroscopy (EDS) confirmed the presence of a protective film of OFM on the wear track, albeit inconsistently deposited. Although the adsorption of the OFM on the metal surface was observed to be dependent on the chemical reactivity of the functional groups, levels of unsaturation, and hydrocarbon chain length of the OFM, the frictional performance was not always directly correlated with the surface coverage and tribofilm thickness. This implies that the friction reduction mechanism can involve other localized processes at the interface between the metal surface and lubricant oil. The occasional variation in friction observed for these OFMs can be attributed to the stability and durability of the boundary film formed during the rubbing phase.
\end{abstract}

Keywords: organic friction modifiers; friction; wear; film thickness

\section{Introduction}

Growing concerns regarding climate change caused by greenhouse gas emission as well as the need for improved energy efficiency in automotive engines have led to a shift toward the use of low-viscosity lubricants [1]. Although low-viscosity lubricants contribute to improved energy efficiency by reducing fluid friction, they can have a detrimental impact on the boundary and mixed lubrication regimes where friction and wear at the contact interfaces are particularly high. Therefore, conventional lubricating oils (transmission fluids, engine oils, etc.) are formulated with a variety of additives for minimizing the parasitic energy loss and for achieving the desired performance under different lubrication regimes. Friction modifiers (FMs) are an important class of lubricant additives for reducing friction when there is no sufficient liquid at the contact interface [2].

Typically, the FMs used in engine oil formulations belong to the class of organomolybdenum compounds and organic friction modifiers (OFMs). The former type is mostly based on sulfur- and phosphoruscontaining compounds that can produce hazardous lubricant discharge consisting of sulfated ash, phosphorus, and sulfur (SAPS). With the tightening of environmental regulations in recent years, OFMs (primarily made up of carbon, hydrogen, oxygen, and nitrogen) are starting to replace the former

* Corresponding author: Febin CYRIAC, E-mail: febin_cyriac@ices.a-star.edu.sg 
compounds, thereby minimizing SAPS discharge.

OFMs are amphiphilic surfactant molecules having a nonpolar aliphatic tail group of at least 10 carbon atoms attached to the polar head group [3]. It has been proposed that OFMs form a film on the surface with the polar head groups adhering to the metal surface, while the hydrocarbon tails are oriented perpendicular to the surface and remain solubilized in the base oil $[4,5]$. These amphiphilic molecules form a vertically oriented, closely packed monolayer due to dipole-dipole interactions between adjacent polar head groups of the adsorbed OFM molecules and van der Waals forces between the hydrocarbon chains. A multilayer cluster is formed by molecular stacking on the adsorbed OFM monolayers [6]. Although friction reduction in the presence of OFMs is generally ascribed to the formation of monolayer film on the contact surface $[4,7]$, some studies have confirmed the formation of viscous layer deposits on the metal surface ranging between 10 and $100 \mathrm{~nm}$ thickness [8-10]. The films are strong and can withstand high pressure because of the cumulative van der Waals forces between the molecules. Under the application of load and shear, the adsorbed layers, which are difficult to compress, can easily slip between the opposing polar end groups, leading to reduced friction and adhesion [3].

OFMs can be of diverse chemistries, including esters, alcohols, amines, amides, imides, carboxylic acids, phosphates, borates, and their derivatives [2]. A number of structural factors influence the effectiveness of the OFMs. For instance, molecules with long aliphatic chain lengths can affect the packing density and thickness of the adsorbed film [3-5]. Strong films are formed on the contact surface when the chain lengths of the OFMs are similar to that of the base oil [6]. The increased polarity of the head groups with higher intermolecular hydrogen bonding capability is also critical for enhanced surface interactions and reduced friction [7]. In addition, the properties (e.g., polarity) of the base oil, the presence of other lubricant additives and/or contaminants in the base oil, the characteristics of the metal surface, and the engine temperature can influence the performance of OFMs [8,9]. Antiwear and extreme pressure additives added to the base oil, as well as contaminants formed by oxidative degradation of the lubricants, can sometimes have an antagonistic effect on the friction-modifying characteristics of OFMs. Lower temperatures are favorable for efficient adsorption of OFM molecules on the surface, whereas higher temperatures can lead to decreased tenacity and desorption of the OFM tribolayers [10]. Increasing the concentration of OFMs in the base oil does not always lead to improved frictional performance. As much as $1 \%$ OFM concentration has been shown to be cost effectively in reducing friction, but only a marginal improvement in friction is usually observed above a concentration of $1 \%$ [8].

The performance of OFMs in mineral oil base stocks has been widely studied [11-15]. However, to the best of our knowledge, studies aimed at understanding the performance of OFMs in polar base oils have not been widely reported. Unlike polyalphaolefin and mineral oils, polyol ester oil, a Group V base oil, is likely to exhibit a high affinity toward the steel surface because of its polarity. As a result, the OFMs present in the oil may have to compete with the ester molecules to adsorb on the metal surface [16]. In this context, this study aimed to determine the mechanism of action of OFMs in polar ester oil-particularly the influence of structural factors on the generation of tribofilms for reducing friction and wear. Tribological measurements were performed using a mini traction machine (MTM) at a temperature of $130{ }^{\circ} \mathrm{C}$. The effectiveness of different OFMs in forming a protective boundary film was studied at zero speed (to circumvent hydrodynamic lubrication) using the three-dimensional (3D) spacer layer imaging method (SLIM) - an in situ imaging technique available with the MTM. Wear track formation on the disk specimens was analyzed ex situ using both scanning electron microscopy (SEM) coupled with energy dispersive X-ray spectroscopy (EDX) and a 3D surface profiler. The combined analysis of the data obtained from the tribological and surface characterization techniques provided insights into the tribofilm formation mechanism. 


\section{Materials and methods}

\subsection{Materials}

Reagent grade amphiphiles (Fig. 1) purchased from Sigma Aldrich were used as model OFMs. Stearamide, oleamide, stearic acid, elaidic acid, and linolenic acid were used to study the effect of the molecular structure of the hydrocarbon tail on OFM performance. Stearic acid, stearamide, and stearamine were used to gauge the influence of the OFM head group. Stearic acid, arachidic acid, and behenic acid were selected to study the effect of the length of the hydrocarbon tail on OFM performance. A NSF HX-1 approved low deposit forming polyol ester, with a viscosity of $20 \mathrm{~mm}^{2} / \mathrm{s}$ at $40{ }^{\circ} \mathrm{C}$ and $4.4 \mathrm{~mm}^{2} / \mathrm{s}$ at $100{ }^{\circ} \mathrm{C}$ (viscosity index 140 , cloud point $-39{ }^{\circ} \mathrm{C}$, pour point $-51{ }^{\circ} \mathrm{C}$ ), was donated by Croda Singapore Pte Ltd. and used as the base oil.

Lubricant oil formulations were prepared by dissolving $0.5 \mathrm{wt} \%$ of the OFM in ester oil at $60{ }^{\circ} \mathrm{C}$ under stirred conditions (except for stearamide, which dissolved at $110{ }^{\circ} \mathrm{C}$ ). Considering that some of the OFMs (arachidic acid, behenic acid, stearamine, and stearamide) precipitated when the oil solutions were cooled to room temperature $\left(23^{\circ} \mathrm{C}\right)$ at time intervals ranging from $30 \mathrm{~min}$ to $24 \mathrm{~h}$, tribological measurements were performed immediately after sample preparation. Except for stearamide, no precipitation was observed in all the samples during the cooling phase to room temperature after each tribological test.

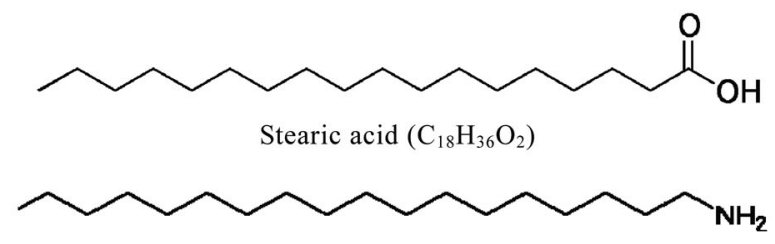

Stearamine $\left(\mathrm{C}_{18} \mathrm{H}_{39} \mathrm{~N}\right)$

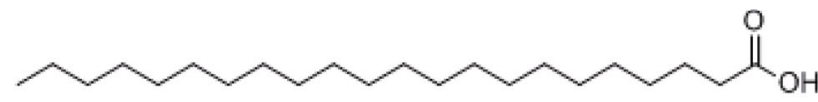

Arachidic acid $\left(\mathrm{C}_{20} \mathrm{H}_{40} \mathrm{O}_{2}\right)$

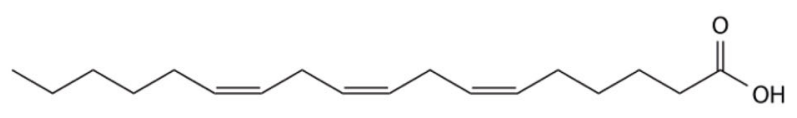

Linolenic acid $\left(\mathrm{C}_{18} \mathrm{H}_{30} \mathrm{O}_{2}\right)$

\subsection{Tribological characterization}

Tribological measurements were made using an MTM obtained from PCS Instruments, UK (Fig. 2). In this test, a steel ball is loaded against a steel disk, each driven independently by servo motors that enable a combination of different rolling and sliding speeds. The temperature can be varied from ambient to $150{ }^{\circ} \mathrm{C}$ and controlled precisely using a closed-loop system. Traction is measured using a force transducer mounted on the ball shaft and can be operated under elastohydrodynamic lubrication (EHL), mixed, and boundary lubrication regimes. The MTM is equipped with a SLIM 3D surface mapper for measuring the thickness of the boundary additive film formed on the steel ball. The film thickness is measured to nanometer accuracy by the principle of optical interferometry. During the film thickness measurement, the ball and disk are stopped momentarily and automatically reverse loaded against the mapper disk. Then, the system periodically captures the interference image of the static Hertzian contact between the steel ball and the mapper disk with the help of a high-resolution red-green-blue color camera. The film thickness map of the circular contact area is determined (using a stand-alone analysis program) by matching the color in the image to a calibration file.

The properties of the ball on the flat disk geometry (AISI 52100 Steel) and the test conditions are summarized in Tables 1 and 2, respectively. Before

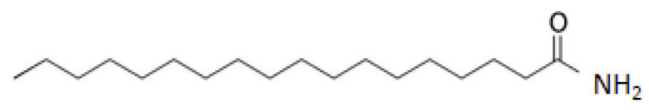

Stearamide $\left(\mathrm{C}_{18} \mathrm{H}_{37} \mathrm{NO}\right)$

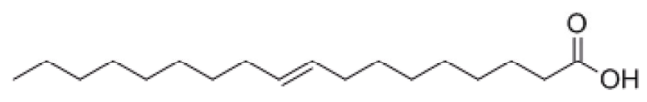

Elaidic acid $\left(\mathrm{C}_{18} \mathrm{H}_{34} \mathrm{O}_{2}\right)$
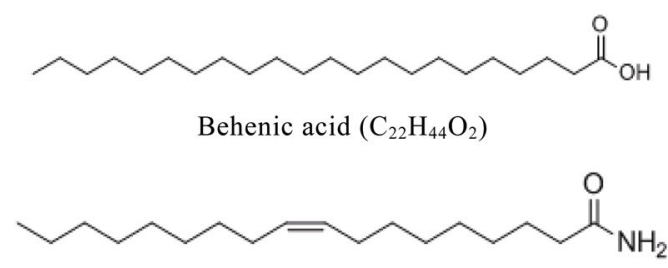

Oleamide $\left(\mathrm{C}_{18} \mathrm{H}_{35} \mathrm{NO}\right)$

Fig. 1 Chemical structures of OFMs employed in the study. 


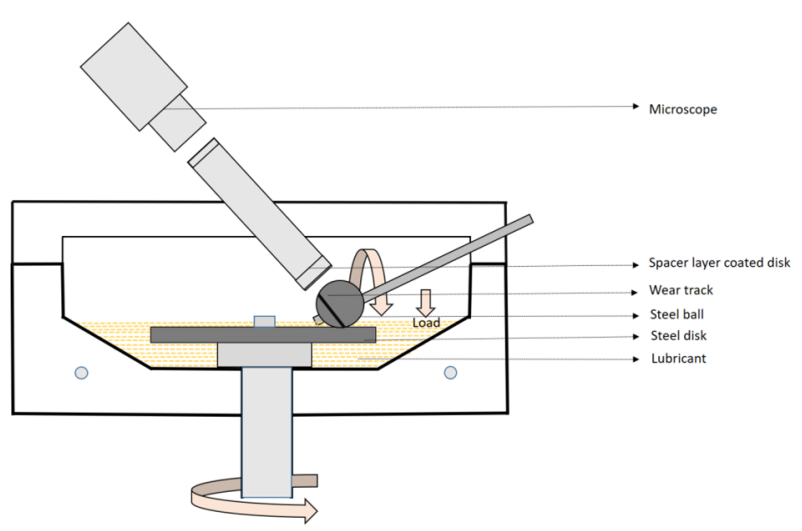

Fig. 2 Schematic of the MTM with SLIM option.

the test, the test specimens were ultrasonically cleaned with ethanol and acetone and dried using nitrogen gas.

Tribological measurements were made in three distinct phases (Table 2). First, the ball and disk were rubbed at an entrainment speed of $0.1 \mathrm{~m} / \mathrm{s}$ at a slide roll ratio (SRR) of $100 \%$ to generate the tribofilm of the OFM. Under these conditions, the contact operated in the mixed lubrication regime. This was followed by tribofilm measurement using the 3D SLIM. Finally, Stribeck curve measurements were taken in the speed range between 0.001 and $2 \mathrm{~m} / \mathrm{s}$. Because data acquisition from the lowest to the highest speed contributed to higher boundary friction (Fig. 3), possibly due to the damage of tribofilms formed during the rubbing phase, Stribeck curve measurements were instead performed from the highest to the lowest speed. The coefficients of friction (CoFs) reported are the average values of two measurements. The Stribeck curves were found to be reproducible with no significant difference in the tribofilm thickness measured using SLIM.

\subsection{Surface characterization}

\subsubsection{SEM-EDX analysis}

The wear track on the steel disk was evaluated using a JEOL JSM-7900F field emission scanning electron microscope with an Oxford INCA EDX spectrometer. The measurements were carried out at a beam voltage of $10 \mathrm{keV}$, and images were acquired at $160 \times$ and $3,000 \times$ magnifications. The metal disk was rinsed in $n$-hexane to remove the base oil before SEM-EDX analysis.

\subsubsection{Surface morphology analysis}

An Infinite Focus Alicona benchtop optical 3D surface profiler was used to characterize surface roughness and wear scar width on the metal disk after the friction test. The equipment applies the technique of focus variation and can detect topographic features in the nanometer-to-micrometer range. Topographical information is obtained by combining a small depth of focus of an optical system with vertical scanning. The measurements were performed at a vertical resolution of $100 \mathrm{~nm}$ using a 20× objective lens. All the wear measurements were performed on the disk because of its lower hardness compared with the ball and, therefore, higher susceptibility to wear.

\section{Results}

\subsection{Tribological characterization}

The influence of the structural aspects of the OFMs - the presence of unsaturated carbon in the hydrocarbon tail, the difference in the head groups,

Table 1 Properties of the ball on flat disk geometry (AISI 52100 steel).

\begin{tabular}{cccccc}
\hline Geometry & Diameter $(\mathrm{mm})$ & Roughness, $R_{\mathrm{q}}(\mathrm{nm})$ & Elastic modulus $(\mathrm{GPa})$ & Poisson ratio & Hardness $(\mathrm{Hv})$ \\
\hline Ball & 19.05 & 20 & 210 & 0.29 & $800-920$ \\
Disk & 46 & 10 & 210 & 0.29 & $720-780$ \\
\hline
\end{tabular}

Table 2 Test conditions employed in this study.

\begin{tabular}{cccccc}
\hline Phase & Load $(\mathrm{N})$ & $P_{\max }(\mathrm{GPa})$ & Speed range $(\mathrm{m} / \mathrm{s})$ & Slide roll ratio $(\mathrm{SRR})(\%)$ & Temperature $\left({ }^{\circ} \mathrm{C}\right)$ \\
\hline $\begin{array}{c}\text { Preconditioning } \\
\text { Tribofilm }\end{array}$ & 36 & 1.0 & 0.1 & 100 & 130 \\
$\begin{array}{c}\text { measurement } \\
\text { Stribeck curve } \\
\text { measurement }\end{array}$ & 36 & 0.57 & - & 130 & 130 \\
\hline
\end{tabular}




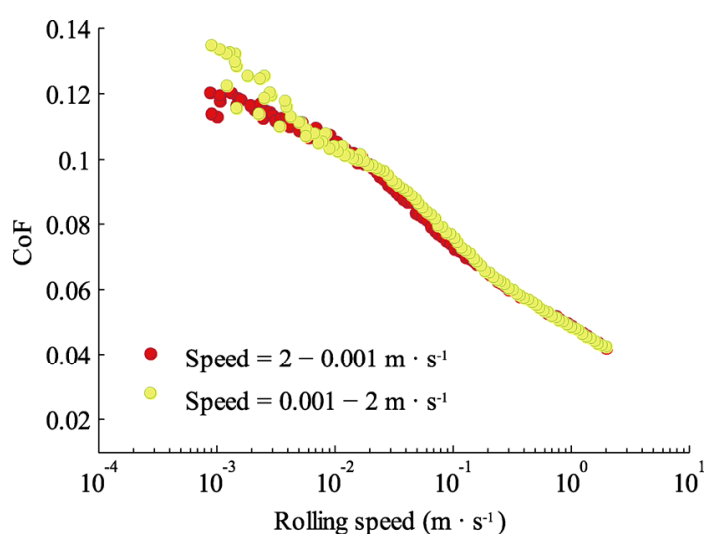

Fig. 3 Influence of ascending and descending speed range on the Stribeck curve.

and length of the hydrocarbon tail - on their lubrication performance was investigated by analyzing the $\mathrm{CoF}$, mean center layer thickness, and film thickness map for the OFMs obtained from the MTM studies. Rheological characterization of the samples performed at $130{ }^{\circ} \mathrm{C}$ using an Anton Paar 702 rheometer with Couette geometry showed that the addition of the OFMs did not influence the bulk properties of the base oil (Fig. 4). Therefore, the observed frictional response can be solely attributed to the structural aspects of the additives.

\subsubsection{Influence of unsaturation}

To elucidate the effect of the unsaturation of the OFM on the frictional behavior, the tribological responses of two fatty acid amides - stearamide and oleamide-were compared. Figure 5 shows interference

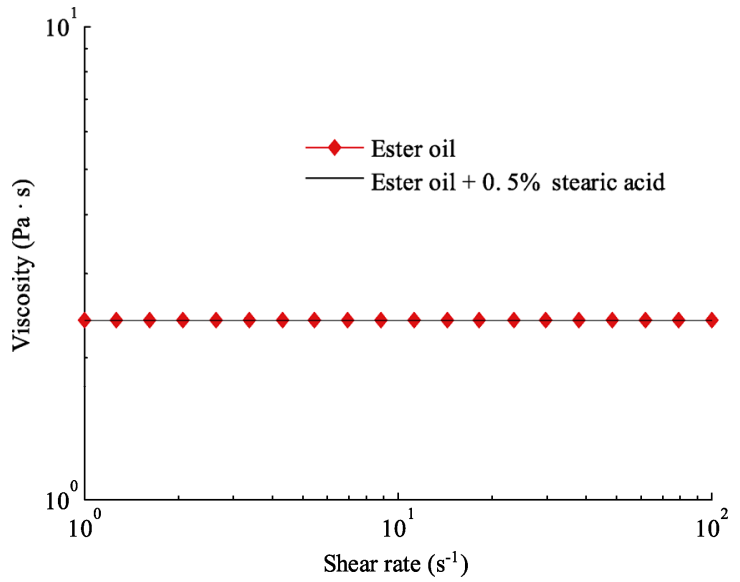

Fig. 4 Viscosity of ester oil measured at $130{ }^{\circ} \mathrm{C}$ with and without additive.

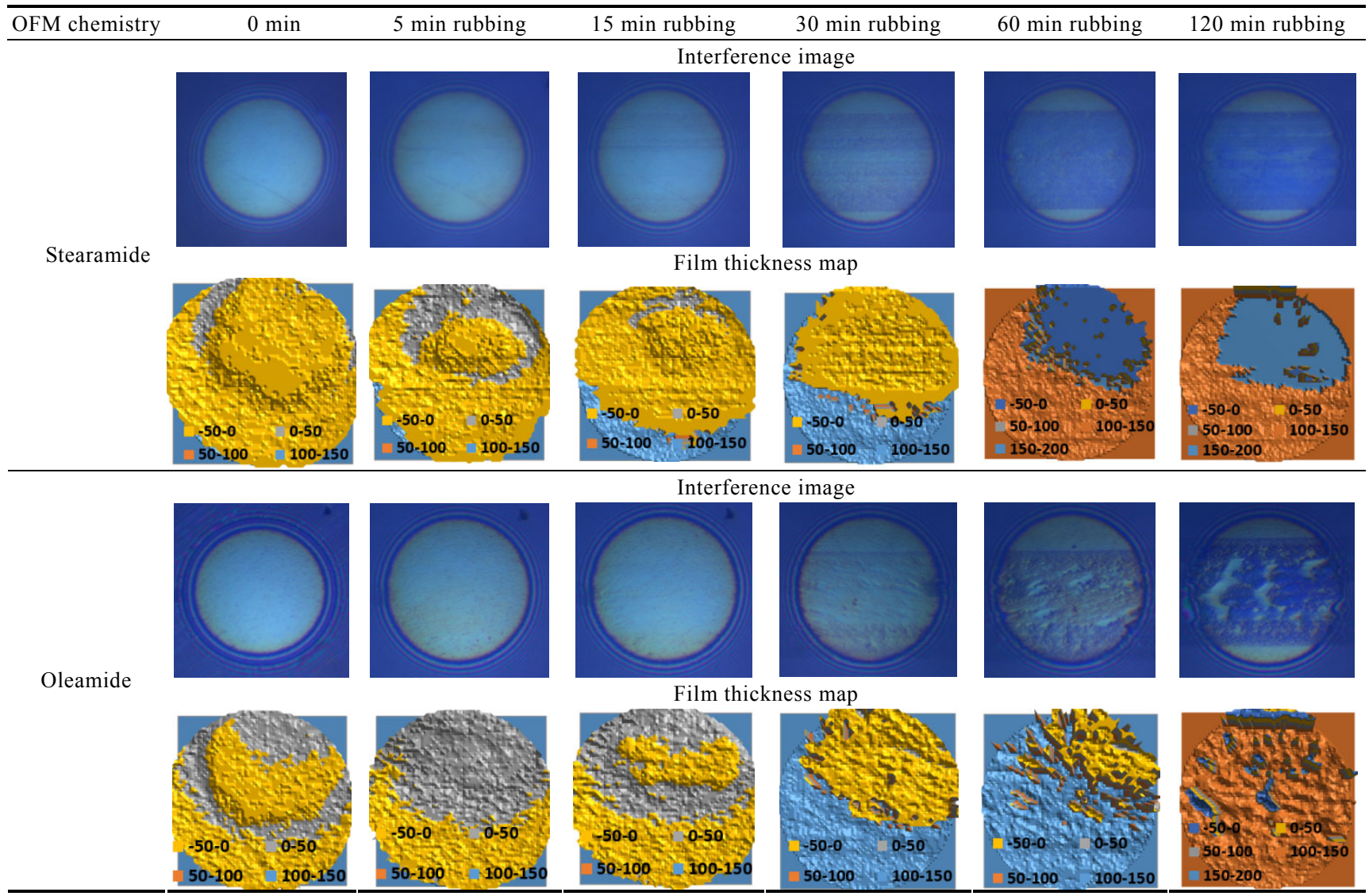

Fig. 5 Interference images and film thickness maps for stearamide and oleamide obtained at different rubbing durations. 
images and the corresponding film thickness maps acquired at different rubbing durations. In all the images, the sliding direction is from left to right. The tribofilm morphology formed by stearamide after $120 \mathrm{~min}$ of rubbing appears to be uniformly distributed, whereas a patchy film is observed in the case of oleamide. The mean layer thickness at the center of the contact calculated from the interference images is shown in Fig. 6(a). It can be seen that the deposition of both the OFM molecules is insignificant

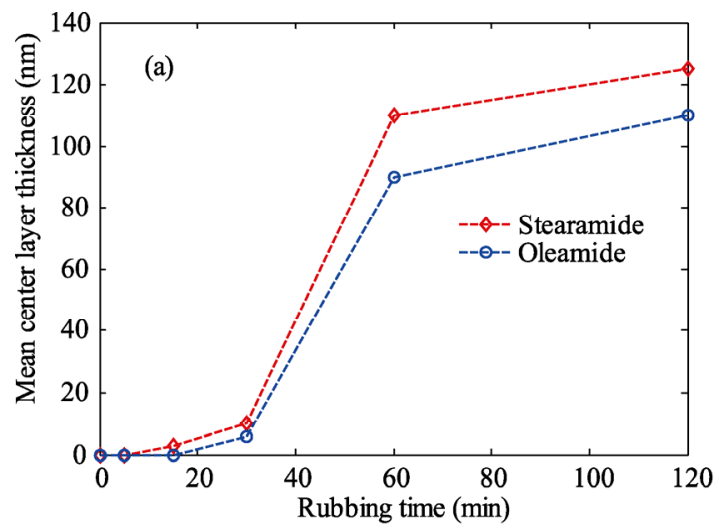

during the initial rubbing phase (0-30 min); subsequently, a thick film formed after $60 \mathrm{~min}$ of rubbing. Stearamide exhibited a thicker uniform film compared with oleamide during extended rubbing. While these results provide insights into the dynamics of OFM deposition, they should be interpreted with caution because the film thicknesses determined at each pixel location across the horizontal profile of the contact had a large variation.

Figures 7(a) and 7(b) show the Stribeck curves

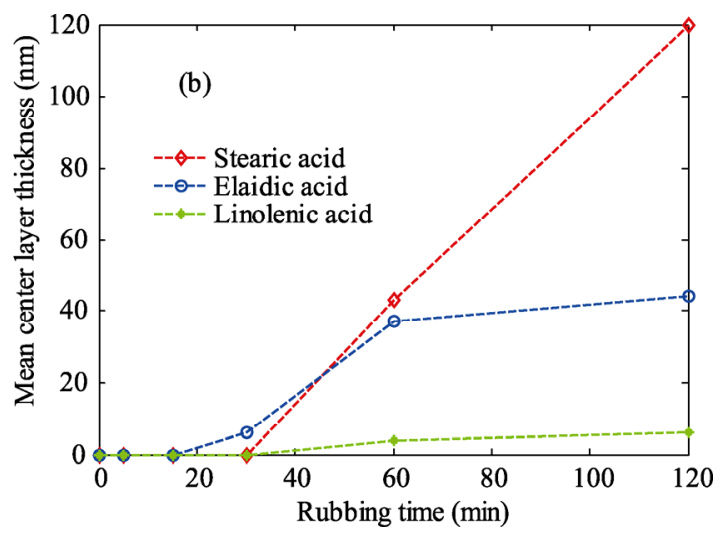

Fig. 6 Mean central layer thickness calculated from interference image for saturated and unsaturated amide and acid molecules.

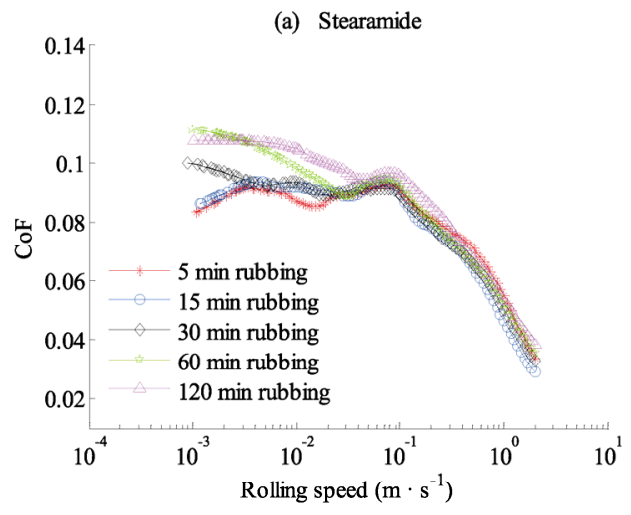

(c) Stearamide

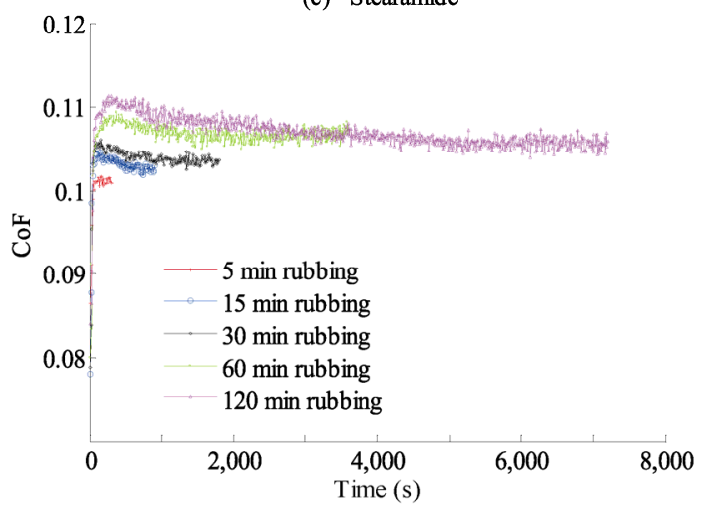

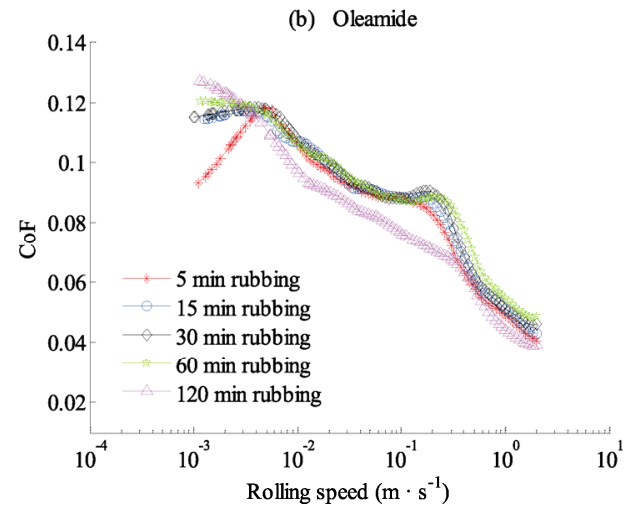

(d) Oleamide

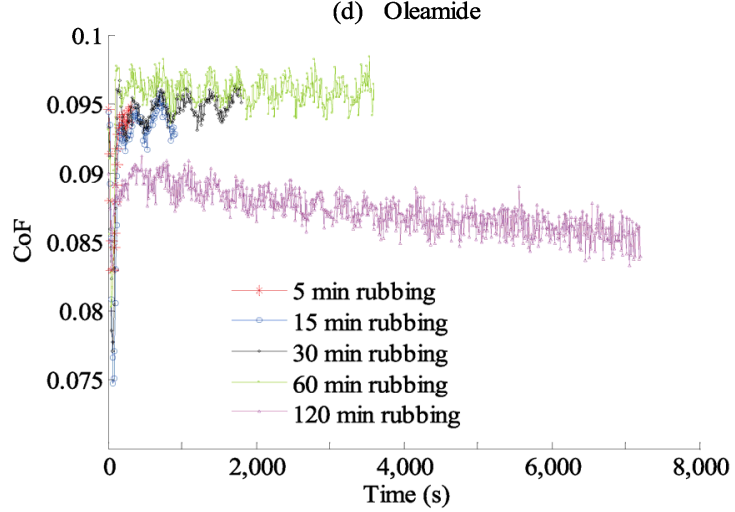

Fig. 7 (a, b) Stribeck curves and (c, d) evolution of CoF as a function of time for stearamide and oleamide. 
for the amides measured after different rubbing durations. In the presence of stearamide, no notable difference in $\mathrm{CoF}$ can be seen in the mixed lubrication regime, which corresponds to a rolling speed ranging from 2 to $0.1 \mathrm{~m} / \mathrm{s}$. However, an increase in the rubbing time resulted in higher friction in the boundary regime $(0.1-0.001 \mathrm{~m} / \mathrm{s})$. The frictional response of oleamide was similar to that of its saturated counterpart, except for the measurements carried out after $2 \mathrm{~h}$ of rubbing (Fig. 7(b)). Herein, prolonged rubbing contributed to lower friction under mixed lubrication conditions.

Friction data recorded during the rubbing phase for the two amides (Figs. 7(c) and 7(d)) correlate well with the Stribeck curve responses. Despite the adsorption layer being thicker in the case of stearamide (Fig. 6(a)), an increase in friction with extended rubbing could primarily be attributed to an increase in surface roughness resulting from accelerated wear. Although this effect did not significantly influence friction under mixed lubrication conditions, the adsorbed stearamide layer might not have withstood harsher conditions prevailing under the boundary regime. Therefore, it is thought that the breaking away of the OFM (stearamide) layer led to an increase in microasperity contact, resulting in higher boundary friction. In contrast, an apparent drop in friction in the case of oleamide at 120 min of rubbing (Fig. 7(d)), despite its lower adsorbed layer thickness, can be attributed to an enhanced self-lubricating property owing to its lower shear strength [17]. At the same time, the enhanced lubricating action observed in the mixed lubrication regime tends to fall in the boundary regime (Fig. 7(b)). This behavior could be caused by the instability of the adsorbed oleamide layer under harsher rubbing conditions.

The Stribeck curves for stearamide and oleamide obtained at different rubbing durations are compared in Fig. 8. Correlating the frictional response of the OFMs with their tribofilm thicknesses reveals that a thicker film (formed by stearamide) generally contributes to lower boundary friction. Given that a lower friction is usually achieved when complete coverage of OFM is observed [18], the higher friction observed in the presence of oleamide in the boundary lubrication regime could be partly caused by a patchy heterogeneous film formed by the OFM. In comparison with stearamide, oleamide has a single carbon double bond arranged in cis-configuration, resulting in a bent shape for the alkyl chain (Fig. 1). This implies that, unlike its saturated counterpart, oleamide cannot adopt a linear conformation [16, 19, 20]. In turn, this could prevent oleamide from forming a close-packed structure on the metal surface. While this reasoning helps to understand the difference in the lubrication performance of the two OFMs, further experimental evidence is required to confirm this mechanism.

Interestingly, in the boundary lubrication regime, ester oil alone offers better lubricity than in the presence of oleamide. This result can be explained on the following basis. Notwithstanding the stronger interaction of the ester oil with the metal surface owing to its high polarity, oleamide competes with the ester molecules to adsorb on the metal surface. However, the oleamide layer could desorb under low-speed conditions because of its low shear strength, and thus increasing microasperity contacts and resulting in higher friction.

The influence of unsaturation on the frictional
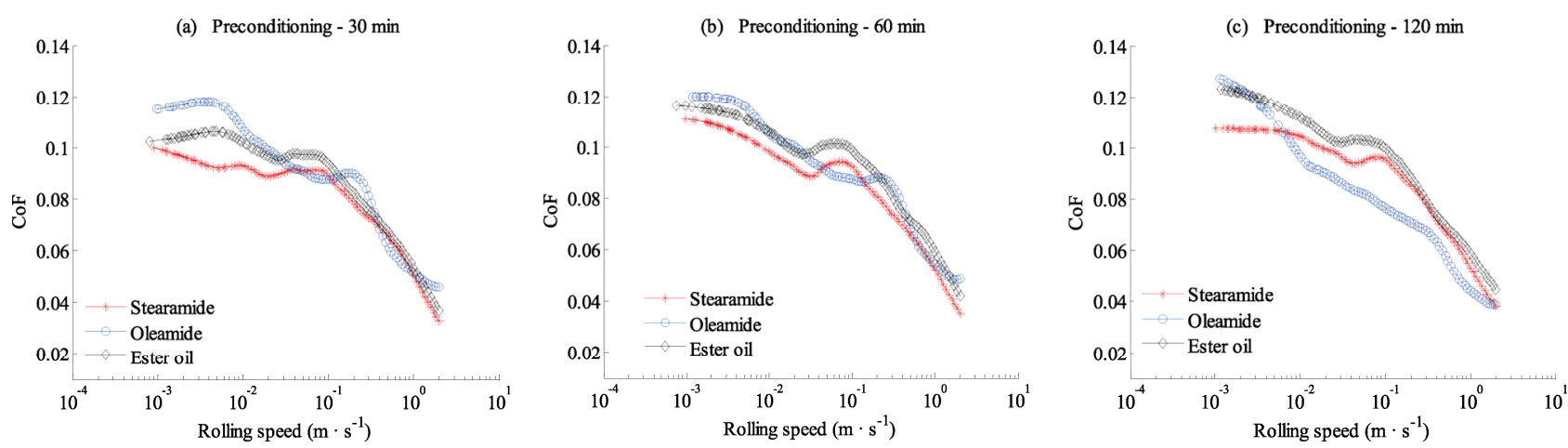

Fig. 8 Influence of rubbing time on the evolution of Stribeck curves for stearamide and oleamide. 
behavior of OFMs was further studied by comparing the tribological performance of long-chain carboxylic acids with zero (stearic acid), one (elaidic acid), and three (linolenic acid) unsaturated bonds, respectively. The interference images obtained during the traction studies (Fig. 9) clearly show differences in tribofilm morphology formed by the three carboxylic acids. While stearic acid and elaidic acid show gradual film buildup with rubbing, the adsorption film formed by linolenic acid is negligible, even after $60 \mathrm{~min}$ of rubbing. The mean center layer thickness at the center of the Hertzian contact calculated from the interference images is shown in Fig. 6(b). Elaidic acid and linoleic acid exhibited thinner films compared with stearic acid. However, the film thickness determined at each pixel location across the horizontal profile of the contact for elaidic acid showed patches of the film reaching up to $160 \mathrm{~nm}$.

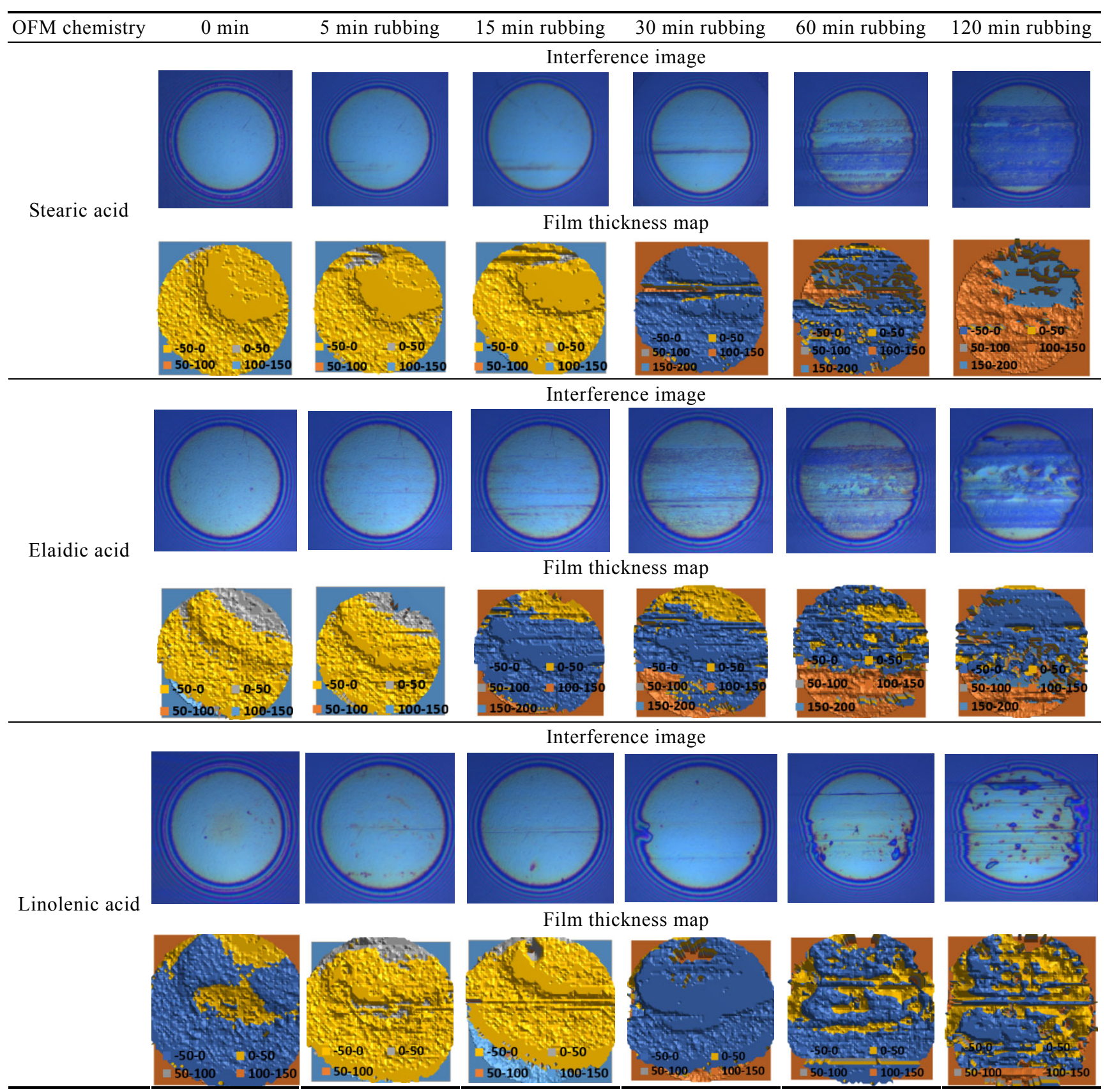

Fig. 9 Interference images and film thickness maps for stearic acid, elaidic acid, and linolenic acid for different rubbing durations. 
Stribeck curves for the carboxylic acids obtained at different rubbing times are shown in Figs 10(a)10(c). For all the carboxylic acids, an increased frictional resistance is observed with rubbing time. However, a disparity in the trend is seen for linolenic acid. With longer preconditioning, the CoF decreased in the mixed lubrication regime. This behavior is similar to the trend observed for oleamide previously (Fig. 7(b)). In addition, compared with stearic acid and elaidic acid, linolenic acid is better at reducing friction under mixed lubrication conditions (Figs. 10(d)-10(f)). Linolenic acid has three carbon cisdouble bonds, which is expected to disrupt the close packing of molecules adsorbed on the surface. Previous studies have shown that OFMs with an unsaturated alkyl chain are less surface active and, therefore, result in higher friction [4, 21-23]. The current result, however, is contrary to those findings. At present, the exact mechanism by which linolenic acid caused a reduction in friction during extended rubbing is not known; however, a possible mechanism is proposed. Instead of forming a vertically oriented layer, linolenic acid could interact strongly with the surface via the polar head group and form tilted aggregated structures. These structures might undergo shear-induced tensile stretching to form a thin film, with the molecules, aligned parallel to the surface. In retrospect, there could be limited interpenetration of the adsorbed linolenic acid layers on the opposing sliding surfaces. Thus, the adsorbed layer (a few nanometers thick) could act as an "antiblocking" agent and thereby reduce asperity interactions on the counter surfaces.

In the case of elaidic acid, the boundary friction coefficient (BFC) evolved nonmonotonously (Fig. 10(b)). This frictional response can be caused by the formation of a disordered, weakly bound structure of the OFM that is not strong enough to withstand the harsher contact conditions at lower rolling speeds. Interestingly, the minimum value of friction $\left(F_{\min }\right.$ "kink") shifted toward the right of the Stribeck curve with the increase in rubbing duration. This phenomenon could result from the breaking away of the adsorbed layer during extended rubbing, leading to an increase in microasperity contact (surface roughness). Because this phenomenon is not apparent for the rest of the OFM chemistries, it is presumed that the change in surface topography, shear, and deformation properties of the FMs and their orientation with respect to the rolling direction could influence

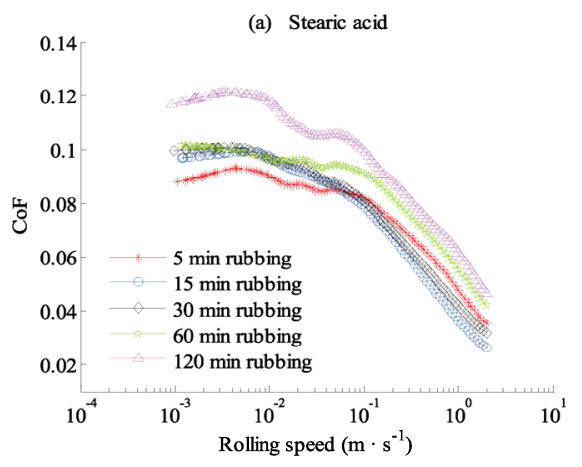

(d) Preconditioning - $30 \mathrm{~min}$

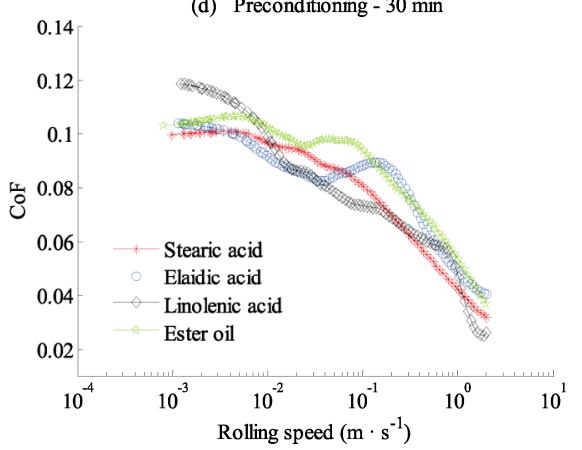

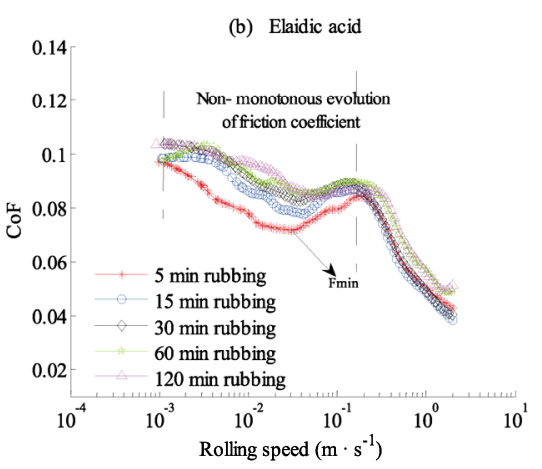

(e) Preconditioning - $60 \mathrm{~min}$

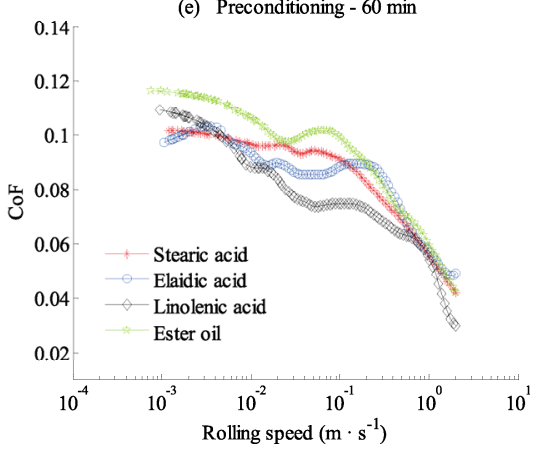

(c) Linolenic acid

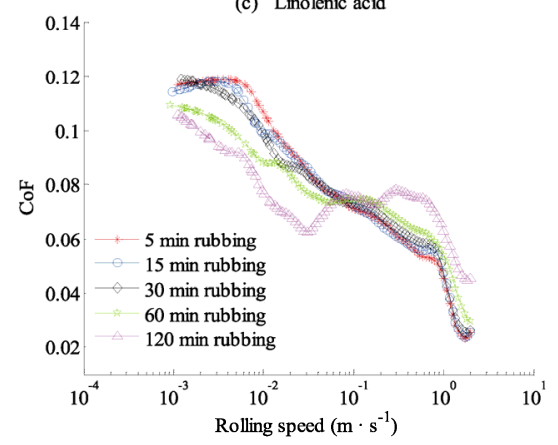

(f) Preconditioning - $120 \mathrm{~min}$

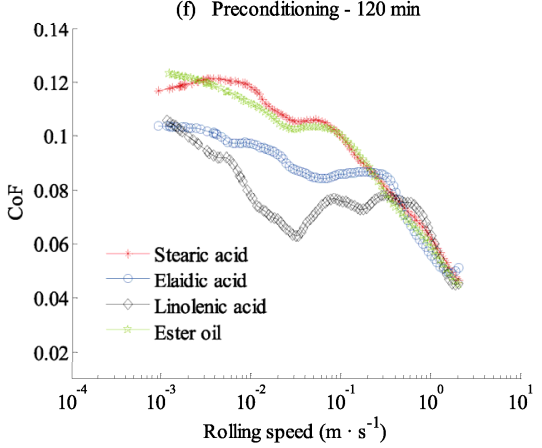

Fig. 10 (a-c) Stribeck curves for long chain carboxylic acids and (d-f) influence of rubbing time on the CoF. 
the tribological performance.

From a structural standpoint, elaidic acid has a double bond in transconfiguration; therefore, it may adopt a linear configuration akin to that of stearic acid [22, 24]. With similar molecular structures, although elaidic acid is expected to have a comparable effect on the friction, it exhibited a lower CoF than stearic acid. The interference images and film thickness map (Fig. 9) show that stearic acid produced an almost complete coverage on the surface during preconditioning, whereas elaidic acid showed nonuniform coverage on the surface. Previous study has shown that molecules with saturated alkyl chains form close-packed monolayer surfaces, leading to a lower friction-speed response [7]. In view of this, the difference in the friction-speed responses observed for the two structurally similar carboxylic acids can be explained as follows. Although stearic acid initially formed a uniform layer on the metal surface, it is likely that the film cannot withstand high pressure at the asperity conjunctions and could have been progressively removed at lower speeds. This may be the reason why stearic acid exhibited a frictional response comparable to that of the base fluid (Fig. 10(f)), and also why elaidic acid shows a better frictional response despite exhibiting a nonuniform surface coverage.

Comparison of Stribeck curves for the carboxylic acids subjected to different rubbing durations shows that, under mixed lubrication conditions, the friction reduced with the increase in the number of unsaturated bonds (Figs. 10(d)-10(f)). This may be due to the presence of multiple reactive centers in the unsaturated molecules that promote mechanochemical deposition of the film on the counter surfaces [25]. Furthermore, under prolonged rubbing, a higher level of unsaturation in the chain contributed to a substantial reduction in friction compared with its saturated counterpart. However, from the current data, it is difficult to ascertain unambiguously the influence of the degree of unsaturation on the boundary friction during the initial phase of rubbing. This can be attributed to partial removal of the protective film formed by the polar species on the surface during the transition from a mixed to a boundary regime.

\subsubsection{Influence of head group}

Figure 11 shows the series of interference images obtained at the center of the contact for stearic acid, stearamide, and stearamine. These images provide insights into the dynamics of film formation. In the case of stearic acid, the film developed with rubbing at a slower rate (film deposition can be seen only after $60 \mathrm{~min}$ ). An accelerated film formation was observed for stearamine (film started to appear approximately after $5 \mathrm{~min}$ of rubbing). For stearamide, the film growth started after $30 \mathrm{~min}$ of rubbing, which is intermediate between stearic acid and stearamine. With time increasing, the tribofilms continued to increase in thickness for all the OFMs, as is evident from the mean central layer thickness calculated from the interference images (Fig. 12(a)). However, the deposited films were found to be of nonuniform thickness, which can be seen from the interference image and film thickness map of the Hertzian contact.

The Stribeck curves obtained for the OFMs with different head groups (Figs. 13(a)-(c)) show an increase in $\mathrm{CoF}$ with rubbing duration. In general, the deposition of the OFMs with different polar head groups led to a reduction in boundary friction of the base fluid. The average BFC for the base fluid was measured to be 0.12 . Accordingly, a decrease in BFC from 0.12 to 0.1 suggests a positive effect on friction reduction for all the three additives. However, among the additives, stearamine displayed the lowest friction under the boundary regime. Stearamine also yielded the highest reduction in friction in the mixed lubrication regime, whereas only a marginal influence can be seen for stearamide and stearic acid.

The friction reduction ranking after $120 \mathrm{~min}$ of rubbing, when there is full surface coverage of the OFM, follows the order: amine > amide > carboxylic acid. The friction reduction properties of OFMs depend on three key interactions: dispersive interaction between the hydrocarbon tail (associated with van der Waals forces), dipole-dipole interaction between the polar head groups, and adhesive bonding between the adsorbate and contact surface [20]. Keeping in view that the hydrocarbon chain structure is the same for all the OFMs, the difference in frictional performance can be attributed to the difference in the polar moiety and, in turn, its influence on the interaction with the substrate. Because tribofilm 


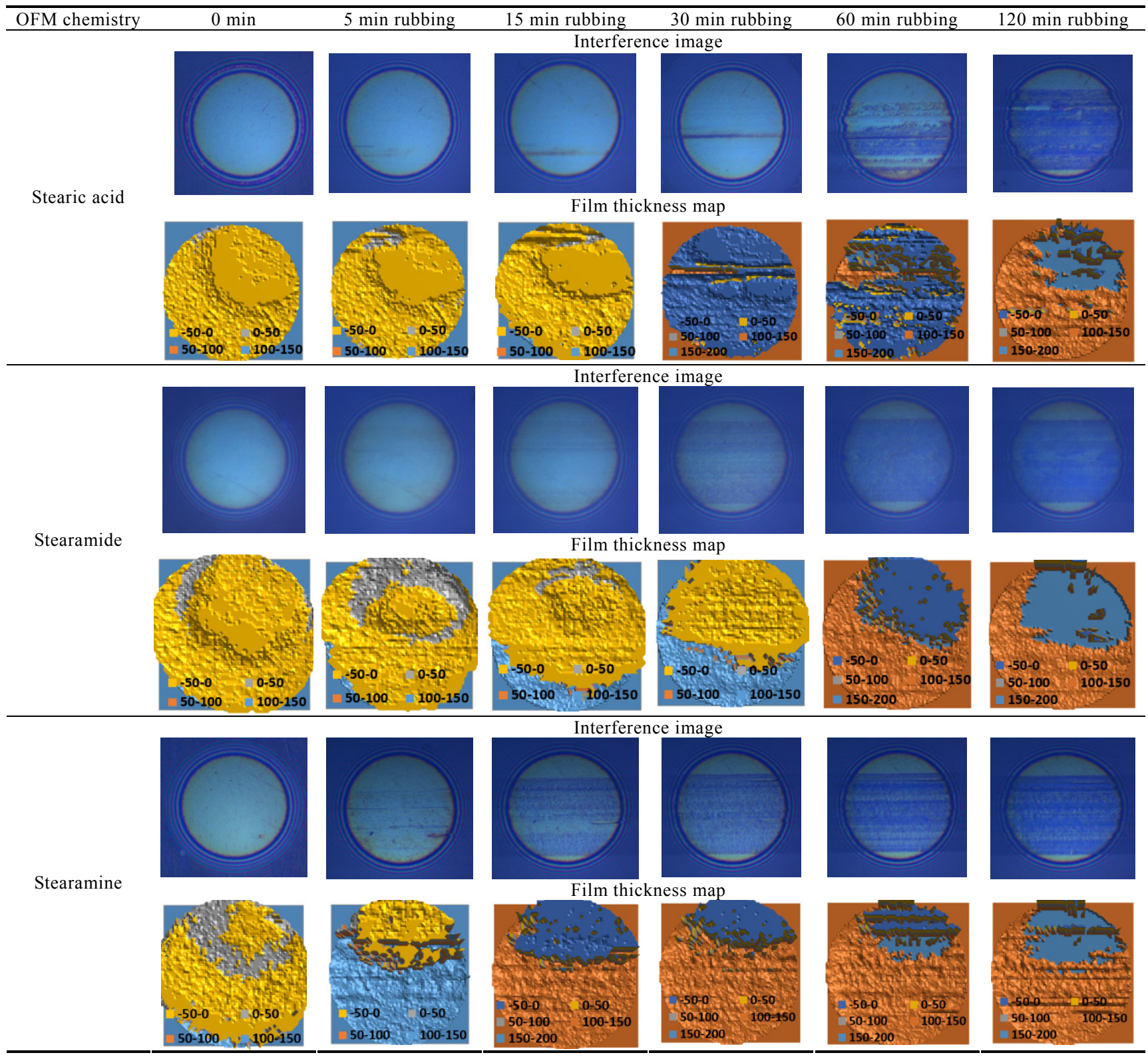

Fig. 11 Interference images and film thickness maps of OFMs with different head groups for different rubbing durations.
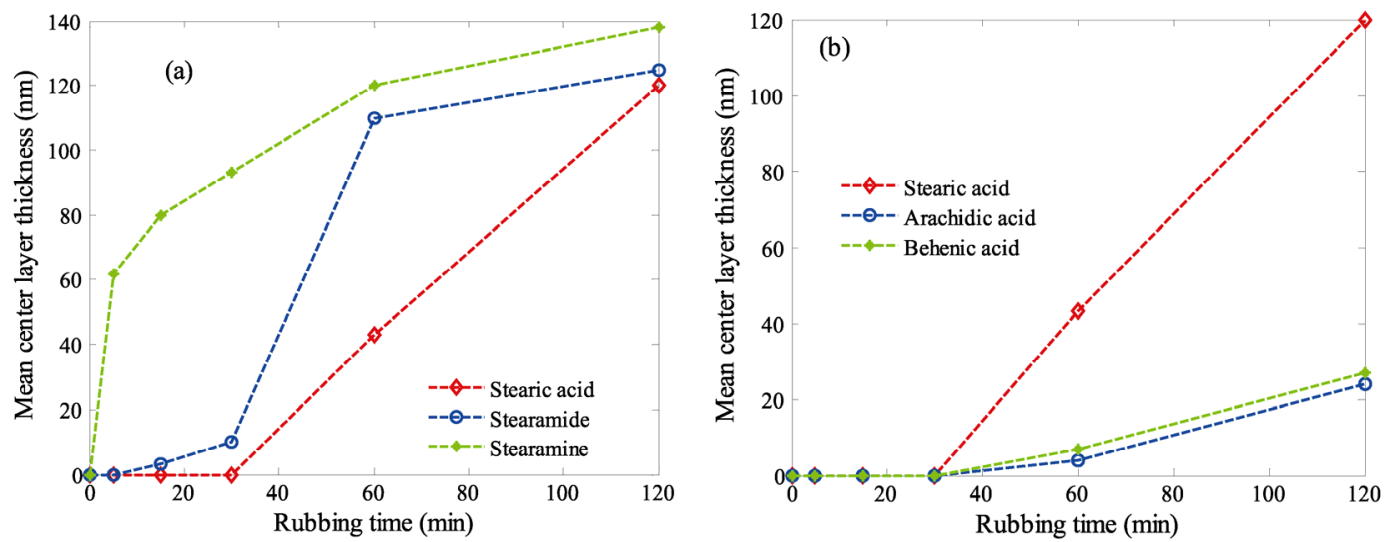

Fig. 12 Mean central layer thickness calculated from interference image for (a) OFMs with different head groups and (b) OFMs with different hydrocarbon chain lengths. 


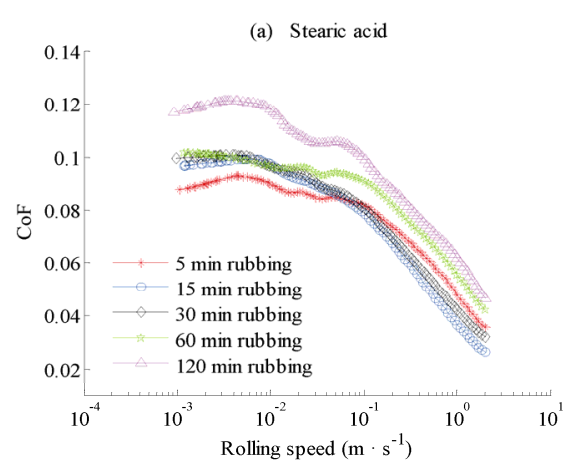

(d) Preconditioning - $30 \mathrm{~min}$

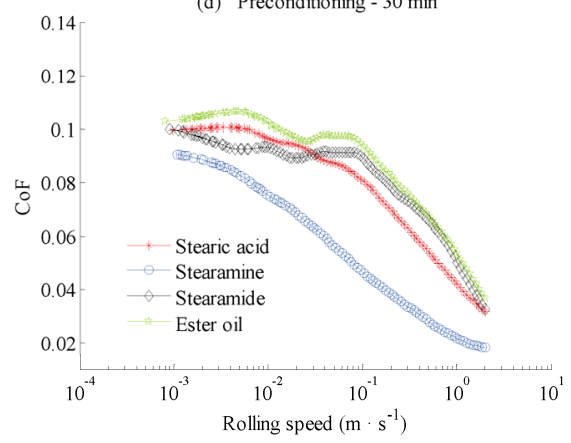

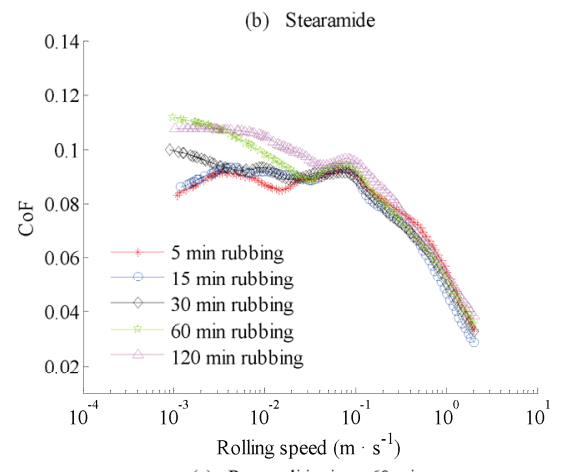

(e) Preconditioning $-60 \mathrm{~min}$

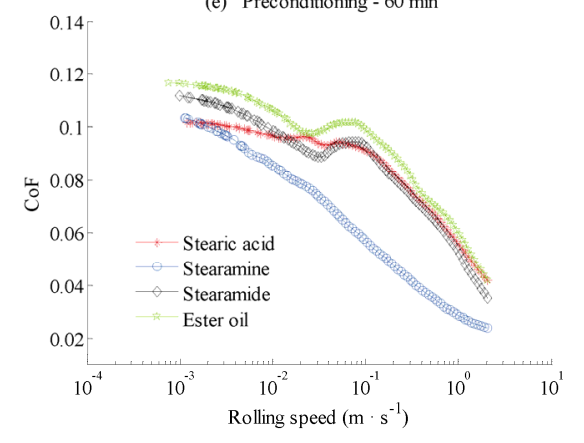

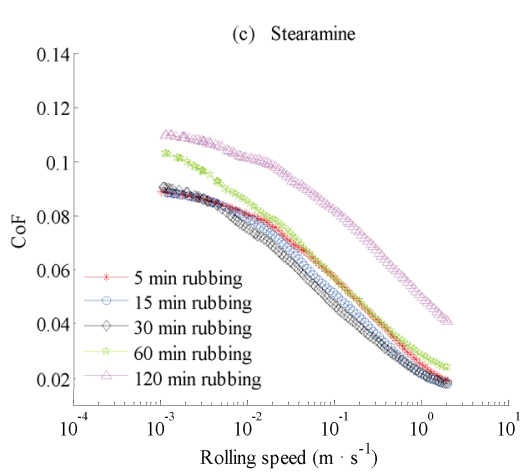

(f) Preconditioning $-120 \mathrm{~min}$

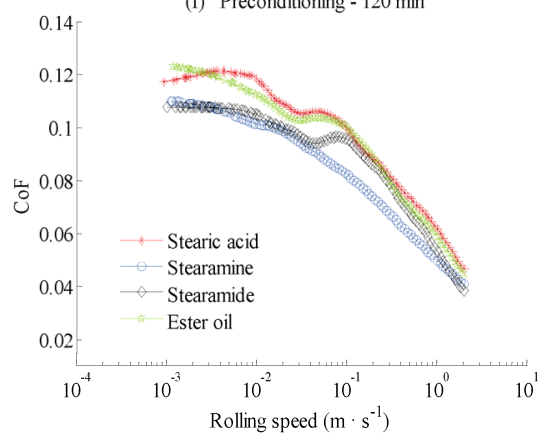

Fig. 13 (a-c) Stribeck curve and (d-f) influence of rubbing time on the CoF for OFMs with different polar moieties.

formation is a dynamic process, the interplay between the kinetics of OFM deposition and removal could also affect metal-to-metal contact [19]. The polarity of the OFMs is in the order of amide > carboxylic acid $>$ amine [26, 27]. If polarity influences the frictional performance, it can be envisaged that stearamide, which has the largest dipole moment, should have a stronger interaction with the surface. Thereafter, the friction should be reduced the most. In contrast, the experimental data show that stearamine is better for reducing friction. Therefore, the mechanism underpinning friction reduction by the OFMs cannot solely depend on the polarity of the head group. However, the presence of nitrogen binding sites in the amide and amine OFMs could influence the orientation, providing tenacious surface adsorption and film durability compared with the carboxylic acid group.

\subsubsection{Influence of length of hydrocarbon tail}

Figure 14 shows the dynamics of film growth for OFMs with different hydrocarbon chain lengths when subjected to different rubbing durations. The results show that a longer rubbing time is needed (as compared with the amine- and amide-based OFMs) to facilitate the adsorption of these OFMs.
This may be the characteristic feature of OFMs with carboxylic acid as the head group. In comparison with stearic acid, arachidic acid and behenic acid displayed only a fractional surface coverage, even after extended rubbing (120 min). This is well captured by the mean center layer thickness obtained from the horizontal profile analysis of the contact (Fig. 12(b)). As noted earlier, the horizontal profile analysis shows a large variation in the film thickness. Intriguingly, the long chain molecules (arachidic acid and behenic acid) formed a much thinner film than stearic acid did. However, the reason for this behavior is not well understood.

A notable increase in CoF was observed for the homologous series of OFMs with increasing rubbing time (Figs. 15(a)-15(c)), despite the formation of thicker films during extended rubbing. As previously stated, this response can be attributed to an increase in microasperity interactions resulting from wear, which masked the positive effect of the OFM on frictional performance. Figures 15(d)-15(f) show the influence of the hydrocarbon chain length of the OFM on friction for different rubbing durations. The friction reduction by the OFMs in the mixed EHL regime can be ranked in the order behenic acid $\left(\mathrm{C}_{22}\right)>$ arachidic acid $\left(\mathrm{C}_{20}\right)>$ stearic 


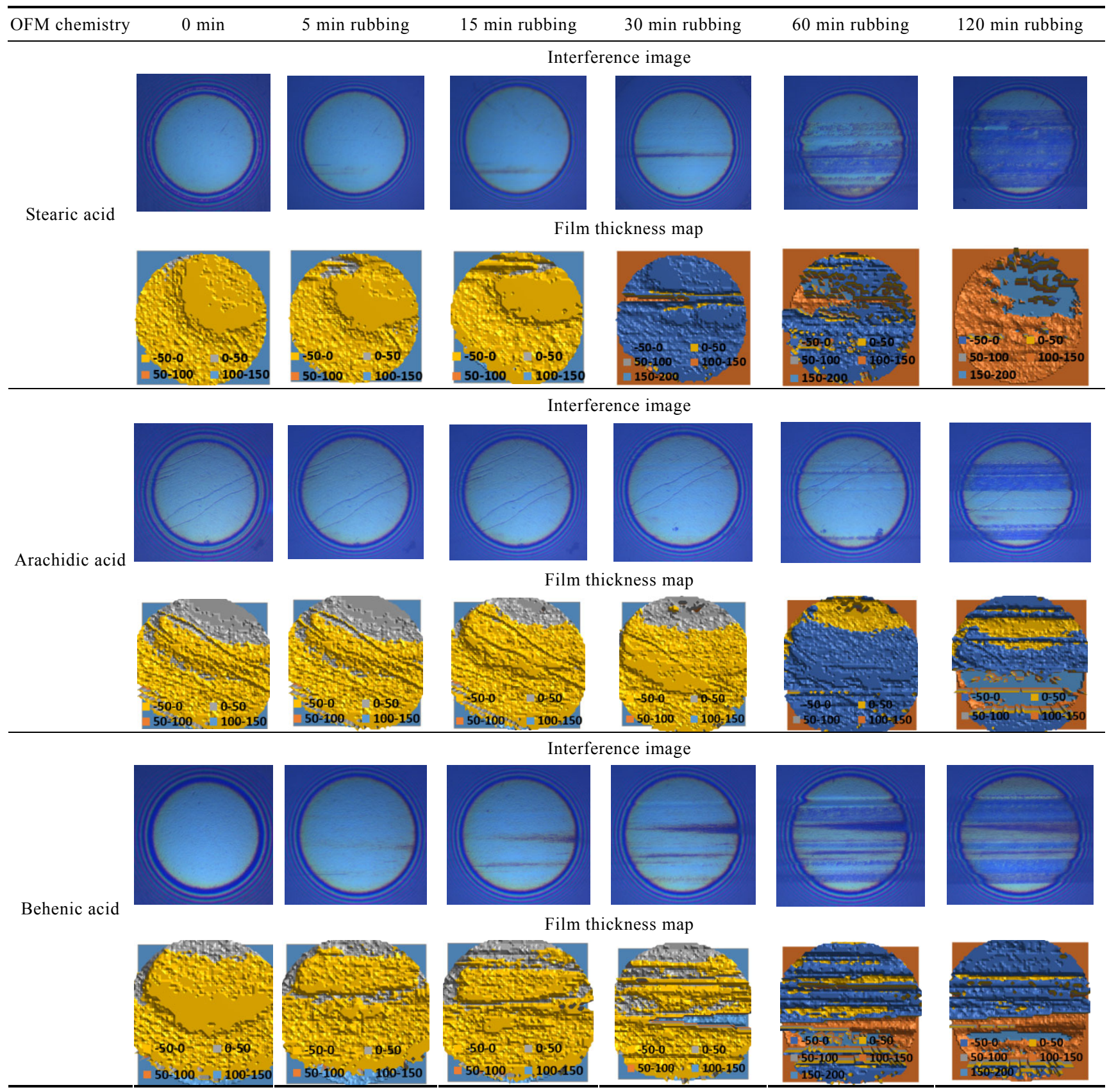

Fig. 14 Interference images and film thickness maps of OFMs with different chain lengths for different rubbing durations.

acid $\left(\mathrm{C}_{18}\right)$. This result is in good agreement with the published literature, where a reduction in friction has been reported with increasing carbon number $[4,5,9,28,29]$. However, the frictional data did not correspond to the OFM film thickness measured from optical interferograms. Arachidic acid and behenic acid, which formed thinner films, exhibited better frictional performance. Therefore, the frictional behavior of these molecules cannot be solely explained based on film thickness data. The decrease in friction for molecules with a longer hydrocarbon tail may result from its ability to trap more base oil molecules near the surface. In turn, this could contribute to an increase in asperity separation, and thus lowering surface shear in the mixed lubrication regime [30,31]. In addition, there could be an increase in lateral cohesive interactions for molecules with longer hydrocarbon tails [5, 29]. It has been reported that the long chain molecules resulting from the higher intermolecular chain 

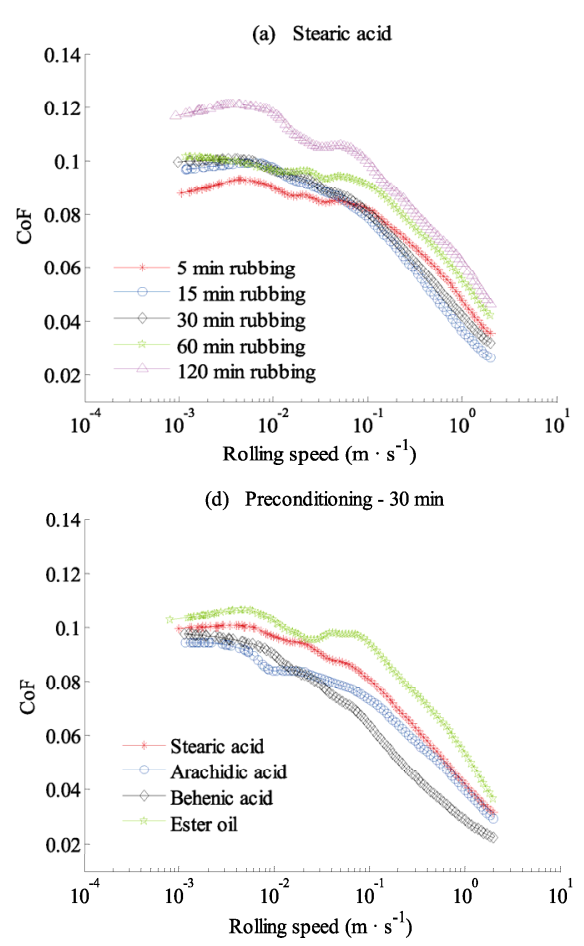

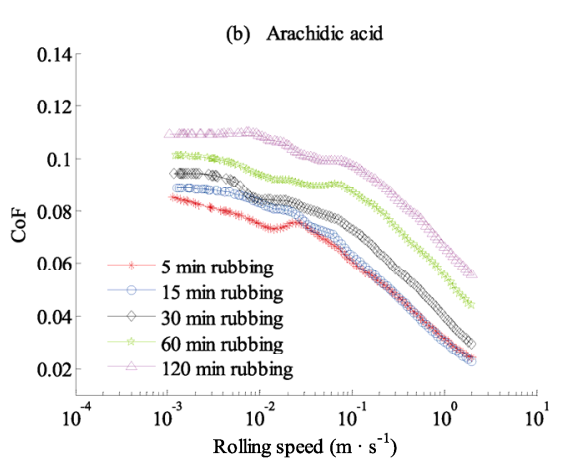

(e) Preconditioning - $60 \mathrm{~min}$

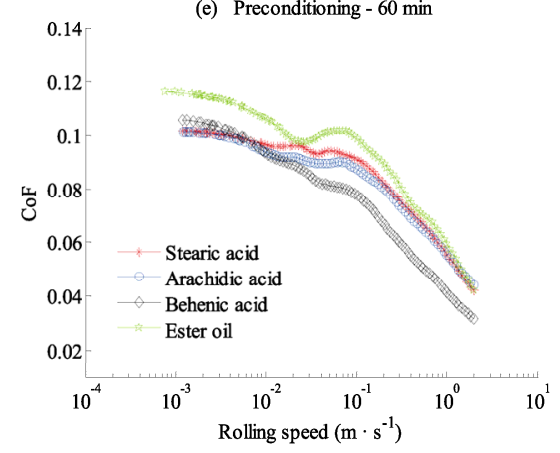

(c) Behenic acid

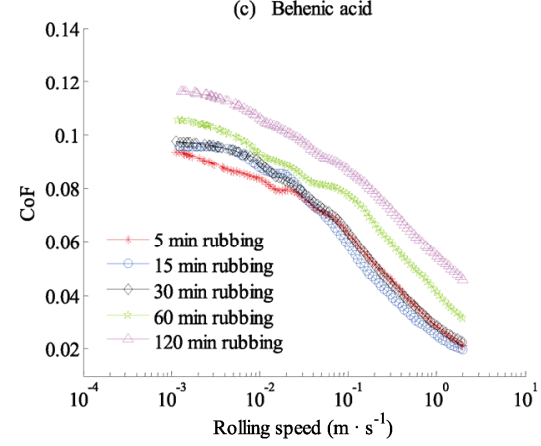

(f) Preconditioning - $120 \mathrm{~min}$

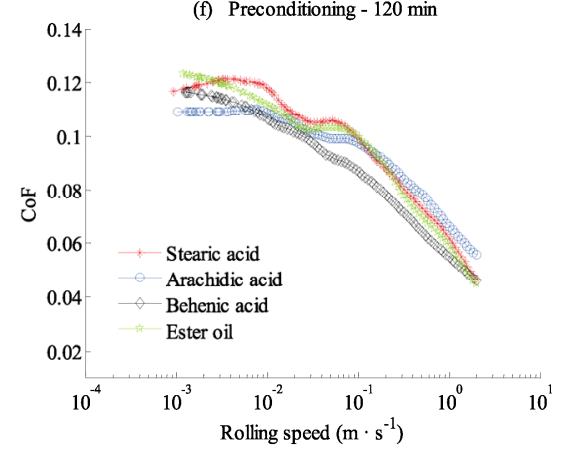

Fig. 15 (a-c) Stribeck curve and $(\mathrm{d}-\mathrm{f})$ influence of rubbing time on the CoF for carboxylic acids with different hydrocarbon chain lengths.

dispersive forces may be more resistant to disruption by shear and asperity contact pressures, leading to reduced wear and friction [5].

However, the influence of additive chain length on boundary friction could not be conclusively established from the experimental results. It could be that these OFMs, which have typically physisorbed onto the surface during rubbing, did not contribute to a resilient boundary film. Therefore, the observed tribological response could be a manifestation of lubrication by patchy films that are not strong enough to prevent penetration by the surface asperities. This means that the intermolecular chain dispersion forces are not sufficient to overcome high-asperity contact pressures experienced in the boundary lubrication regime. This could have negated the role played by the longer hydrocarbon tail in reducing friction.

\subsection{Surface analysis}

\subsubsection{SEM-EDX analysis}

After the tribological tests, the disk specimens were analyzed using SEM-EDX to assess the wear scar and surface reaction films formed on the rolling tracks (Fig. 16). In the case of ester oil alone, a uniform surface smearing is seen along the rolling direction, which implies that the surface was subjected to plastic deformation. The surface morphology also shows thin abrasion grooves and traces of adhesive wear. In comparison, the disk specimens tested in the presence of OFMs displayed notable variation in surface topography within the scar area. For instance, patches of smooth area are observed for stearamine and oleamide, whereas a relatively heterogeneous surface topography is evident for stearic acid and stearamide. Some images show a darker region on the wear track, which may be caused by the deposition of a higher concentration of additives covering the rubbed surface. This theory was later confirmed using EDX analysis.

EDX spectra were collected at the worn surface (positions 1 and 2 in Fig. 16) and regions outside the wear track (position 3 in Fig. 16) to study the chemical state of the frictional surfaces. Figure 17 shows a representative EDX spectrum obtained for linolenic acid. The presence of a significant amount of carbon and oxygen within the wear track can be attributed to the adsorption of OFM on the surface (Table 3). No traces of the additives can be seen 
Ester oil

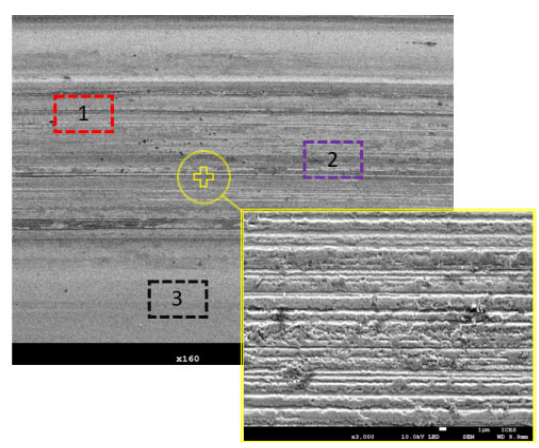

Stearamine

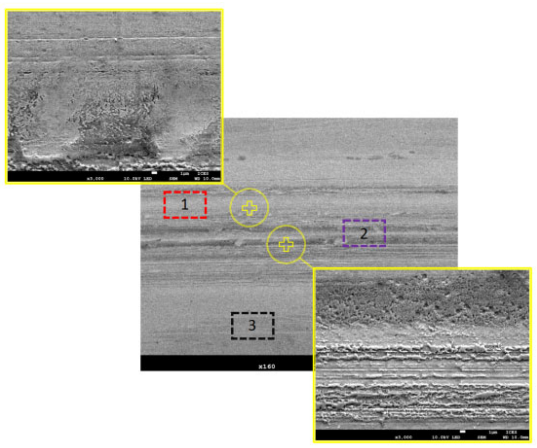

Arachidic acid

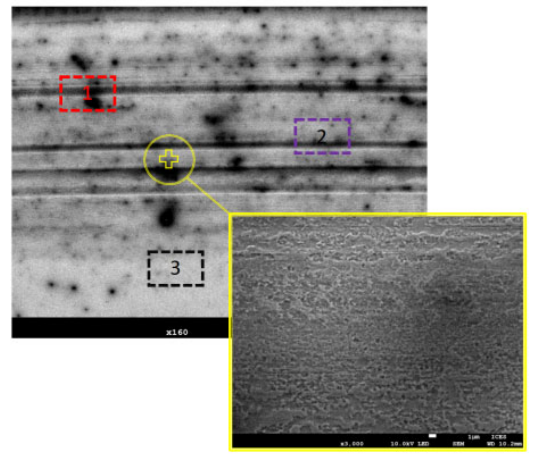

Stearic acid

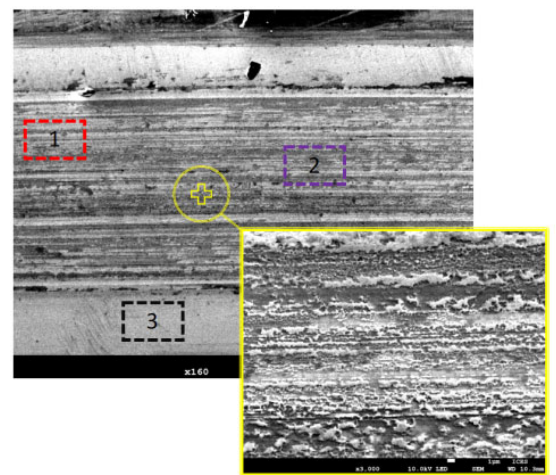

Elaidic acid

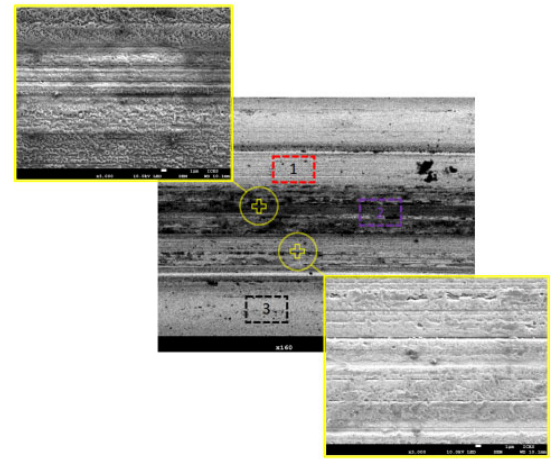

Behenic acid

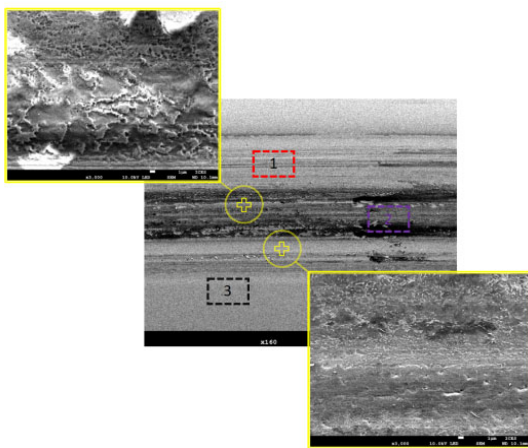

Stearamide

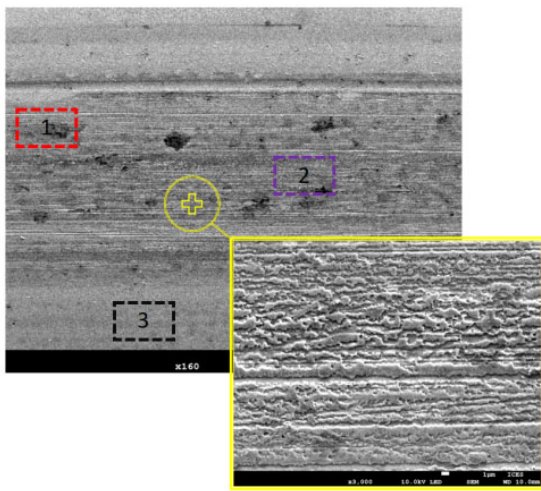

Oleamide

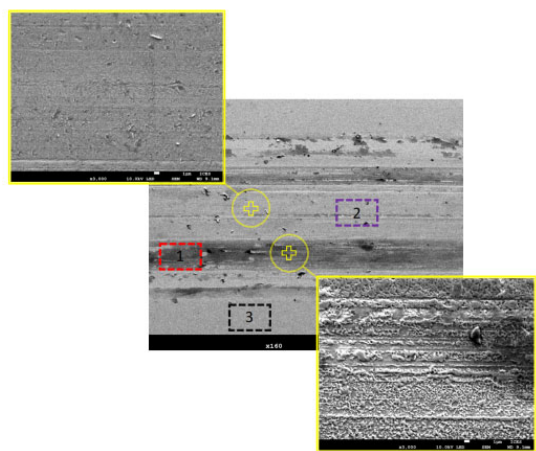

Linolenic acid

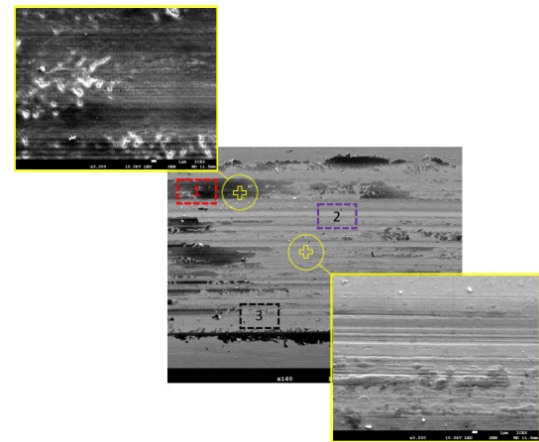

Fig. 16 SEM micrographs of the worn surfaces at $160 \times$ and 3,000× magnification: the numbers show the positions identified for EDX analysis.

outside the wear scar, except for arachidic acid, in which a relatively higher concentration of carbon was detected. These data suggest that a certain amount of shear is a prerequisite for tribofilm formation.

Differences in the concentration of carbon and oxygen within different locations of the wear track can be attributed to inhomogeneous adsorption of the OFMs on the surface. In addition, arachidic acid and linoleic acid showed significantly higher concentrations of carbon and oxygen on the wear track compared with the rest of the additives studied.

\subsubsection{Surface morphology analysis}

To further illustrate the role of OFMs in friction modification, the surface roughness and wear scar width were measured using Infinite Focus Alicona benchtop optical 3D surface measurement equipment. Area-based surface roughness $\left(S_{a}\right)$ measurements were carried out by employing a correlation length of $800 \mu \mathrm{m}$, conforming to the ISO standard.

A representative surface profile and texture analysis of a wear track are shown in Fig. 18. The wear scar width and surface roughness shown in Figs. 19 and 


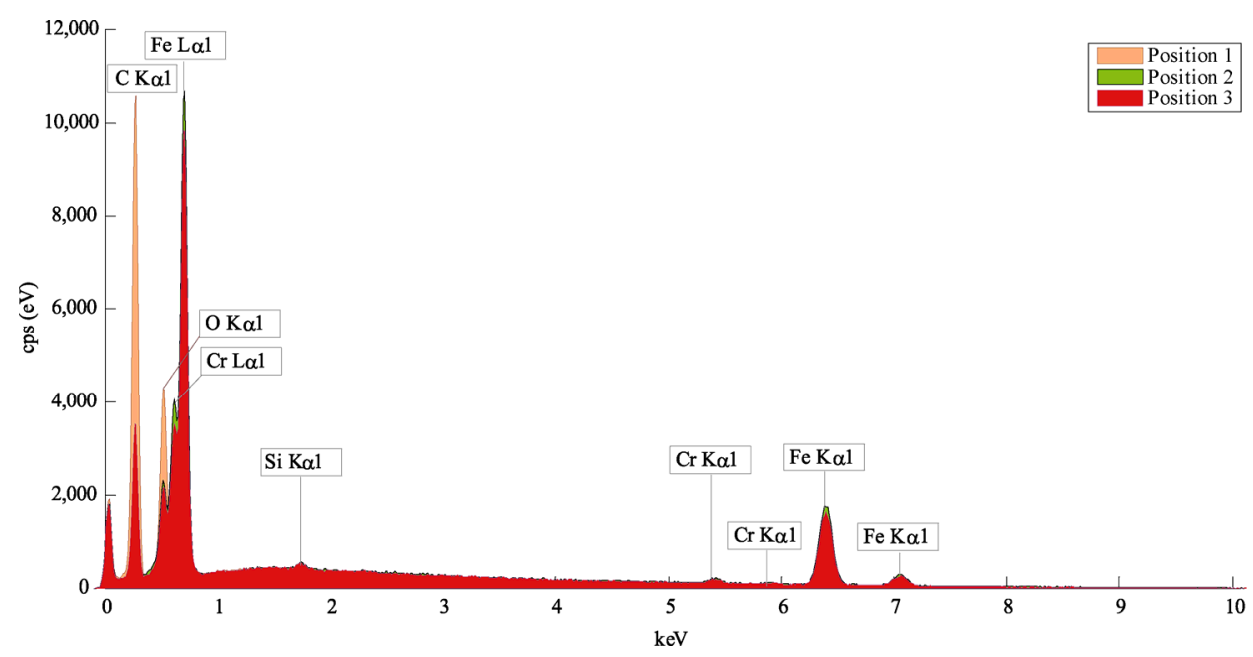

Fig. 17 EDX spectra obtained for linolenic acid at different positions identified in SEM micrographs.

Table 3 EDX data for regions within and outside the wear track.

\begin{tabular}{|c|c|c|c|c|c|c|}
\hline Chemistry & Position & C (\%) & $\mathrm{O}(\%)$ & $\mathrm{Si}(\%)$ & $\mathrm{Cr}(\%)$ & $\mathrm{Fe}(\%)$ \\
\hline & 1 & 6.15 & 7.87 & 0.24 & 1.62 & 84.11 \\
\hline \multirow[t]{3}{*}{ Ester oil } & 2 & 5.89 & 6.76 & 0.21 & 1.83 & 85.31 \\
\hline & 3 & 3.82 & 1.19 & 0.27 & 1.46 & 93.26 \\
\hline & 1 & 7.69 & 8.57 & 0.29 & 2.13 & 81.31 \\
\hline \multirow[t]{3}{*}{ Stearic acid } & 2 & 8.62 & 8.21 & 0.34 & 1.92 & 80.91 \\
\hline & 3 & 3.56 & 1.09 & 0.38 & 1.66 & 93.31 \\
\hline & 1 & 4.17 & 6.27 & 0.25 & 1.8 & 87.52 \\
\hline \multirow[t]{3}{*}{ Stearamide } & 2 & 5.32 & 7.47 & 0.28 & 2.1 & 84.83 \\
\hline & 3 & 3.23 & 1.27 & 0.29 & 1.44 & 93.77 \\
\hline & 1 & 3.89 & 3.55 & 0.28 & 1.73 & 90.56 \\
\hline \multirow[t]{3}{*}{ Stearamine } & 2 & 6.46 & 7.61 & 0.29 & 1.46 & 84.17 \\
\hline & 3 & 4.62 & 1.34 & 0.32 & 1.47 & 92.26 \\
\hline & 1 & 5.42 & 4.78 & 0.24 & 1.81 & 87.74 \\
\hline \multirow[t]{3}{*}{ Elaidic acid } & 2 & 20.32 & 13.42 & 0.24 & 1.94 & 64.09 \\
\hline & 3 & 8.28 & 1.7 & 0.31 & 1.65 & 88.06 \\
\hline & 1 & 4.86 & 3.83 & 0.27 & 1.37 & 89.67 \\
\hline \multirow[t]{3}{*}{ Oleamide } & 2 & 14.48 & 11.56 & 0.23 & 0.19 & 1.66 \\
\hline & 3 & 3.59 & 1.32 & 0.29 & 1.45 & 93.35 \\
\hline & 1 & 34.86 & 14.16 & 0.2 & 1.37 & 49.4 \\
\hline \multirow[t]{3}{*}{ Arachidic acid } & 2 & 39.1 & 13.21 & 0.2 & 1.29 & 46.2 \\
\hline & 3 & 18.38 & 3.09 & 0.26 & 1.47 & 76.81 \\
\hline & 1 & 3.9 & 3.94 & 0.25 & 1.49 & 90.42 \\
\hline \multirow[t]{3}{*}{ Behenic acid } & 2 & 12.6 & 15.26 & 0.2 & 1.82 & 70.12 \\
\hline & 3 & 3.29 & 1.25 & 0.29 & 1.28 & 93.89 \\
\hline & 1 & 36.39 & 10.02 & 0.18 & 1.38 & 52.03 \\
\hline \multirow[t]{2}{*}{ Linolenic acid } & 2 & 14.92 & 3.04 & 0.25 & 1.6 & 80.2 \\
\hline & 3 & 5.06 & 2.73 & 0.27 & 2.16 & 89.78 \\
\hline
\end{tabular}




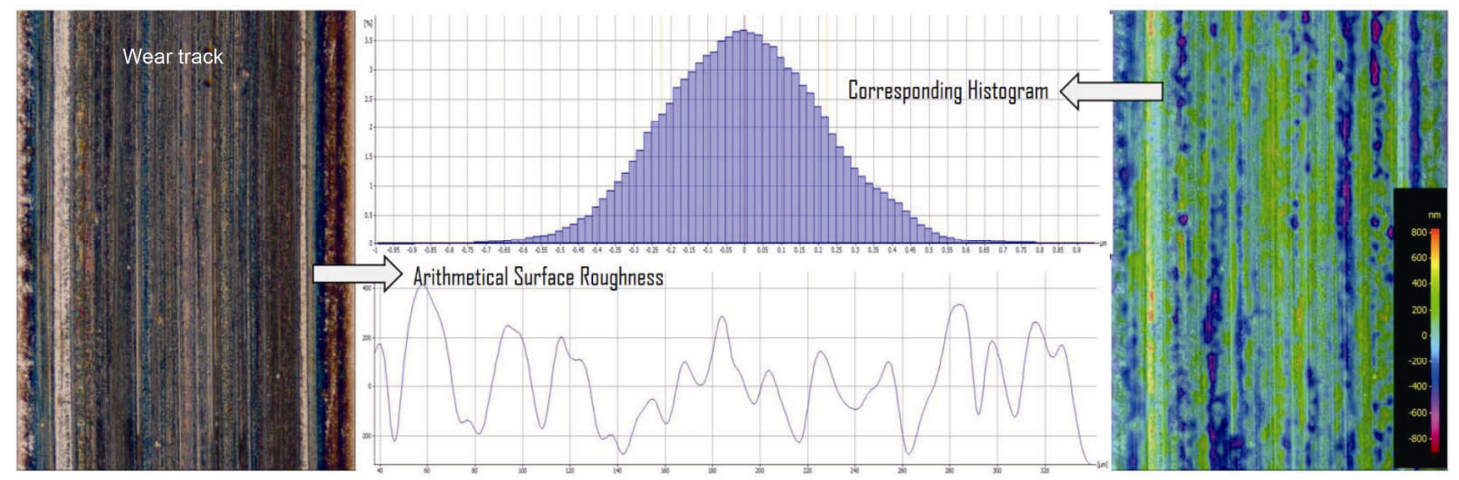

Fig. 18 Profile and surface texture measurement analysis carried out on a worn surface.

20, respectively, are the average values obtained from four random locations along the wear scar. In general, the OFMs with amine and amide functional groups are found to be more effective in reducing wear (narrow wear track and low $S_{\mathrm{a}}$ ) compared with the carboxylic acid OFMs. The improved performance of the former OFM chemistries can be ascribed to the formation of surface reaction films that may have limited material transfer during rubbing. On the other hand, a wider wear track observed for the carboxylic acid OFMs signifies considerable damage imparted to the surface because of an increase

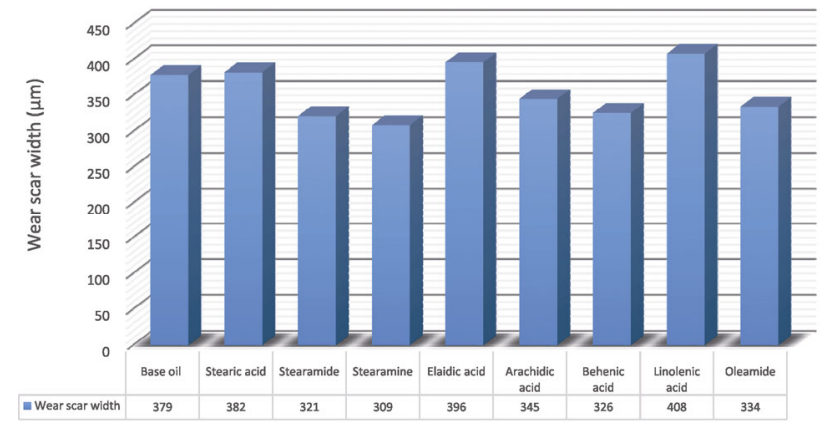

Fig. 19 Average wear scar width measured for different OFM chemistries.

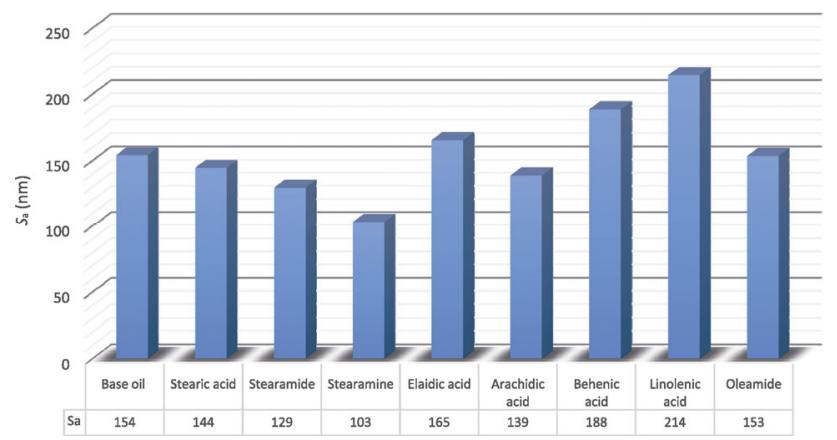

Fig. 20 Average surface roughness measured at the worn track for different OFM chemistries. in plastic deformation. Given that the volume of material worn off from the disk is proportional to the wear scar width to the fourth power, it is inferred that OFMs based on the carboxylic acid group could have contributed to a 1.2 to 3 times increase in wear volume compared with stearamine. In line with this inference, the surface roughness data suggest intensified abrasive wear in the presence of acid-based OFMs. These data support the notion that the chemical reactivity of the functional group of the OFMs plays an important role in reducing wear.

\section{Discussion}

In this study, the influence of structural variables on the tribological performance of OFMs was studied using a number of complementary experimental methods. The changes in friction, surface roughness, worn track width, and chemical composition analysis were used to quantify the lubrication performance of OFMs in polyol ester oil. Our results show that OFMs can mitigate friction to a large extent, even under heavy loads, low sliding speeds, and high temperatures. These conditions are commonly encountered in internal combustion engines between cylinder liners and piston rings. However, not all OFMs were equally effective under all the operating conditions. Some OFMs exhibited comparable frictional response to that of base oil, which was found to be more pronounced under a boundary lubrication regime. Unlike antiwear additives, OFMs may not adhere strongly to the surface; therefore, the films formed are not very durable. As a result, the OFM molecules that are anchored to the metal surface 
can be removed from the surface in the boundary lubrication regime because of its inability to withstand high pressures likely to be present at asperity contacts. Moreover, the interactions responsible for friction reduction are extremely complex, where the film formation depends on the balance between deposition and removal rate. Therefore, the sporadic nature of the friction reduction observed for the OFMs in the boundary regime can be attributed to the stability and durability of the boundary film formed during rubbing.

OFMs based on the carboxyl group usually require a longer rubbing time to diffuse onto the surface for thin-film formation. In contrast, stearamine formed a protective film readily on the surface, which contributed to a much lower friction than those of the amide and carboxyl group OFMs. If polarity was a deciding factor, then the amide having the largest dipole moment would have been strongly adsorbed onto the surface, but that was not the case from the experimental results of this study. Therefore, the adsorption mechanism of OFMs cannot depend solely on the polarity of the functional group. In agreement with the published Refs. [4, 5, 9, 28, 29], a reduction in friction was seen with an increase in the length of the hydrocarbon chain in the mixed lubrication regime. However, the influence of the additive chain length on boundary friction could not be conclusively established from the experimental results. The influence of unsaturation on the frictional performance gave conflicting results. Comparison of Stribeck curves for the carboxylic acids shows a reduction in friction with an increase in the level of unsaturation. However, the frictional performance did not directly correlate with the surface coverage and tribofilm thickness from the interference image. Therefore, film thickness alone cannot account for friction reduction of these OFMs. Therefore, it is likely that other friction reduction mechanisms and localized processes may be involved in influencing the CoF. The presence of multiple reactive centers in the unsaturated molecules might also have played a role [25].

SEM micrographs of the worn surface along the rolling direction show thin abrasion grooves and traces of adhesive wear with varying intensities. For the OFMs studied, the EDX spectra confirmed the presence of an OFM-based protective film on the wear track. However, the elemental analysis displays a significant variation in the concentration of carbon and oxygen, suggesting an incomplete and incoherent tribofilm. The EDX results agree with the optical interference image, which shows nonhomogeneous adsorption. The wear performance of the OFMs was evaluated from the wear scar width and roughness data. The wear scar width was found to be $12 \%$ (stearamine) to $155 \%$ (linolenic acid), higher than the circular contact area diameter of $262 \mu \mathrm{m}$ calculated from the Hertz model. In general, the surfaces lubricated with carboxyl-group-based OFMs showed a larger wear scar and predominant roughening. These were the OFMs that required a longer rubbing time for thin-film formation on the surface. Therefore, it is postulated that a detectable percentage of OFM on the surface, which is a function of chemical reactivity of the functional group, is a prerequisite for reducing wear. Another important observation is an increase in wear scar width with an increase in the level of unsaturation in the carboxylic acids. Because high wear usually results in lower stress in the contact, the conflicting results on the influence of unsaturation on the friction performance may also be associated with the difference in contact conditions.

By considering the BFC, wear track width, and surface roughness measured, the lubrication performance of OFMs in ester oil can be ranked in the order: stearamine $>$ stearamide $>$ arachidic acid $>$ stearic acid $>$ oleamide $>$ elaidic acid $>$ behenic acid $>$ linolenic acid.

Stearamine and stearamide, which formed the thickest films and exhibited better lubrication performance, showed poor solubility in ester oil with high solvation power. Hence, the thick films observed for these additives could be associated with higher additive concentration on the metal surface than in the bulk base fluid. However, this theory could not be corroborated further based solely on the solubility data for the different OFMs. In view of this observation, it is envisaged that the addition of solubility improver to the formulation can help overcome the solubility issues encountered while developing high-performance lubricants. 


\section{Conclusions}

In the present study, we focused on the influence of structural factors on the frictional performance of OFMs. Friction evaluation with film thickness data, wear measurements, and EDX analysis provided insights into their tribological performance. Based on the experimental results, the following conclusions can be drawn:

1) An OFM plays a vital role in mitigating friction in the boundary and mixed lubrication regimes. The reduction in friction is ascribed to the boundary lubrication film containing OFM. EDX spectra confirmed the presence of an incomplete and incoherent OFM-based protective film on the wear track.

2) Some OFMs exhibited a comparable frictional response to that of base oil, which was found to be more pronounced under the boundary lubrication regime. The sporadic nature of the friction observed for these OFMs is attributed to the stability and durability of the boundary film formed during rubbing.

3) Optical interferometry analysis shows the adsorption of OFMs on the metal surface to be heavily dependent on the rubbing duration. Despite the reaction layer being thick, an increase in friction with extended rubbing for the OFMs could primarily be caused by an increase in surface roughness by accelerated wear. This was later confirmed from average area roughness data measured at the worn track using a 3D surface profiler.

4) The frictional performance was found to be a function of the chemical reactivity of the functional group, levels of unsaturation, and hydrocarbon chain length. However, in addition to the influence of structural variables, other friction reduction mechanisms and localized processes may be involved, influencing the CoF.

5) Some additives that displayed better lubrication performance showed poor solubility in ester oil. The result shows that solubility also poses a major challenge in developing top-quality lubricants.

\section{Acknowledgements}

This study was funded by the Agency for Science,
Technology and Research (A*STAR) under a Specialty Chemicals Advanced Manufacturing and Engineering IAF-PP research grant (Grant No. A1786a0026). We thank Yuchan LIU (Singapore Institute of Manufacturing Technology, A*STAR) for her assistance in the surface morphology measurements, and Kwek INEZ and Andrew Shin Boon LIM (Institute of Chemical and Engineering Sciences, $A^{*}$ STAR) for helping with SEM-EDX measurements. We gratefully acknowledge Croda, Singapore, for generously donating the base oil for this study.

\section{References}

[1] Ewen J P, Gattinoni C, Morgan N, Spikes H A, Dini D. Nonequilibrium molecular dynamics simulations of organic friction modifiers adsorbed on iron oxide surfaces. Langmuir 32(18): 4450-4463 (2016)

[2] Tang Z L, Li S H. A review of recent developments of friction modifiers for liquid lubricants (2007-present). Curr Opin Solid State Mater Sci 18(3): 119-139 (2014)

[3] Akhmatov A S. Molecular Physics of Boundary Friction. Jerusalem (Israel): Israel Program for Scientific Translations, 1966.

[4] Jahanmir S, Beltzer M. Effect of additive molecular structure on friction coefficient and adsorption. J Tribol 108(1): 109-116 (1986)

[5] Jahanmir S. Chain length effects in boundary lubrication. Wear 102(4): 331-349 (1985)

[6] Askwith T C, Cameron A, Crouch R F. Chain length of additives in relation to lubricants in thin film and boundary lubrication. Proc R Soc A Math Phys Eng Sci 291(1427): 500-519 (1966)

[7] Davidson J E, Hinchley S L, Harris S G, Parkin A, Parsons S, Tasker P A. Molecular dynamics simulations to aid the rational design of organic friction modifiers. $J$ Mol Graph Model 25(4): 495-506 (2006)

[8] Kenbeek D, Buenemann T, Rieffe H. Review of organic friction modifiers-contribution to fuel efficiency. SAE Technical Paper 2000-01-1792, Paris (France): SAE, 2000.

[9] Castle R C, Bovington C H. The behaviour of friction modifiers under boundary and mixed EHD conditions. Lubr Sci 15(3): 253-263 (2003)

[10] Kenbeck D, Bunemann T. Organic friction modifiers. In Lubricant Additives: Chemistry and Applications. 
Rudnick L R, Ed. Boca Raton: CRC Press, 2009.

[11] Ratoi M, Niste V B, Alghawel H, Suen Y F, Nelson K. The impact of organic friction modifiers on engine oil tribofilms. RSC Adv 4(9): 4278-4285 (2014)

[12] Campen S, Green J H, Lamb G D, Spikes H A. In situ study of model organic friction modifiers using liquid cell AFM; saturated and mono-unsaturated carboxylic acids. Tribol Lett 57(2): 18 (2015)

[13] Choo J H, Forrest A K, Spikes H A. Influence of organic friction modifier on liquid slip: A new mechanism of organic friction modifier action. Tribol Lett 27(2): 239-244 (2007)

[14] Onumata Y, Zhao H Y, Wang C, Morina A, Neville A. Interactive effect between organic friction modifiers and additives on friction at metal pushing V-Belt CVT components. Tribol Trans 61(3): 474-481 (2018)

[15] Okubo H, Watanabe S, Tadokoro C, Sasaki S. Effects of concentration of zinc dialkyldithiophosphate on the tribological properties of tetrahedral amorphous carbon films in presence of organic friction modifiers. Tribol Int 94: 446-457 (2016)

[16] Naveira Suarez A, Grahn M, Pasaribu R, Larsson R. The influence of base oil polarity on the tribological performance of zinc dialkyl dithiophospate additives. Tribol Int 43(12): 2268-2278 (2010)

[17] Briscoe B, Mustafaev V, Tabor D. Lubrication of polythene by oleamide and stearamide. Wear 19(4): 399-414 (1972)

[18] Allan D, Briscoe B J, Tabor D. Lubrication of polythene by oleamide and stearamide-II. Wear 25(3): 393-397 (1973)

[19] Piras F M, Rossi A, Spencer N D. Growth of tribological films: in situ characterization based on attenuated total reflection infrared spectroscopy. Langmuir 18(17): 6606-6613 (2002)

[20] Rudnick L R. Lubricant Additives: Chemistry and

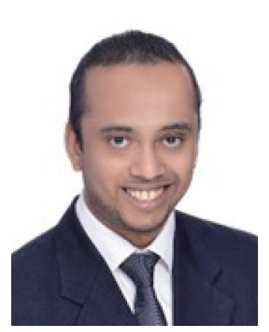

Febin CYRIAC. He received his B.Tech. degree in mechanical engineering from Anna University, India in 2008, and a dual master's degree in rheology with specialization in polymer and materials engineering from University of Huelva, Spain, and University of Minho, Portugal in 2012 (Erasmus Mundus). He gained his Ph.D.
Applications. 3rd edn. Boca Raton (USA): CRC Press, 2017.

[21] Spikes H. Friction modifier additives. Tribol Lett 60(1): 5 (2015)

[22] Campen S, Green J, Lamb G, Atkinson D, Spikes H. On the increase in boundary friction with sliding speed. Tribol Lett 48(2): 237-248 (2012)

[23] Jahanmir S, Beltzer M. An adsorption model for friction in boundary lubrication. ASLE Trans 29(3): 423-430 (1986)

[24] Crespo A, Morgado N, Mazuyer D, Cayer-Barrioz J. Effect of unsaturation on the adsorption and the mechanical behavior of fatty acid layers. Langmuir 34(15): 4560-4567 (2018)

[25] Kuwahara T, Romero P A, Makowski S, Weihnacht V, Moras G, Moseler M. Mechano-chemical decomposition of organic friction modifiers with multiple reactive centres induces superlubricity of ta-C. Nat Commun 10(1): 151 (2019)

[26] Minkin V I, Osipov O A, Zhdanov Y A. Dipole Moments in Organic Chemistry. Boston (USA): Springer, 2012.

[27] LeFèvre R J W. Dipole Moments: Their Measurement and Application in Chemistry. London (UK): Methuen \& Co., Ltd., 1948.

[28] Bowden F P, Leben L. The friction of lubricated metals. Philos Trans R Soc A Math Phys Eng Sci 239(799): 1-27 (1940)

[29] Beltzer M, Jahanmir S. Role of dispersion interactions between hydrocarbon chains in boundary lubrication. ASLE Trans 30(1): 47-54 (1987)

[30] Zhu Y X, Ohtani H, Greenfield M L, Ruths M, Granick S. Modification of boundary lubrication by oil-soluble friction modifier additives. Tribol Lett 15(2): 127-134 (2003)

[31] Zhang J, Meng Y G. Boundary lubrication by adsorption film. Friction 3(2): 115-147 (2015)

degree from University of Twente, the Netherlands, majoring tribology in 2016. He is currently working as a scientist at the Institute of Chemical and Engineering Sciences, A*STAR, Singapore. His research interest covers many aspects of tribology, rheology, and surface characterization techniques. In 2018 he was honored Captain Alfred E. Hunt Memorial Award from STLE for authoring the best paper dealing with the field of lubrication. 


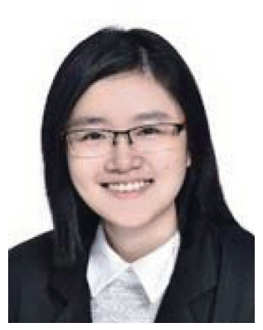

Xin Yi TEE. She received her bachelor of science (honors) with major in chemistry \& biological chemistry at Nanyang Technological University (NTU), Singapore, in 2017. She is currently a research

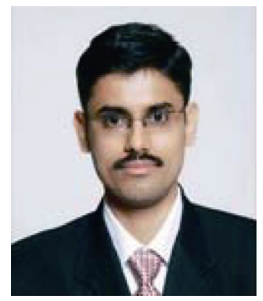

Sendhil K. POORNACHARY. He is a scientist in the Formulated Products Division at the Institute of Chemical and Engineering Sciences, A*STAR. He graduated with a B. Tech. in chemical and electrochemical engineering from Central Electrochemical Research Institute (CECRI), India, and an M.Tech. in chemical engineering

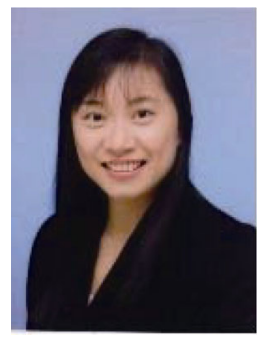

Pui Shan CHOW. She received her B.Eng. degree in chemical engineering from the National University of Singapore in 1996 and a Ph.D. degree in chemical engineering from the University engineer at Institute of Chemical and Engineering Sciences, A*STAR. Her research interests include rheology, tribology, and their application in the formulation of lubricant oils.

from Indian Institute of Technology (IIT), Delhi. He obtained his Ph.D. in chemical and biomolecular engineering from the National University of Singapore (NUS) in 2008. His research interest lies in structure-property relationships in lubricant additives and surface characterization using spectroscopy and neutron/X-ray scattering techniques for correlating adsorption behavior and tribological performance.

of Cambridge, UK, in 2000. She is the team leader of the Formulated Science Team within the Formulated Products Division at the Institute of Chemical and Engineering Sciences, A* STAR. Her research interest lies in the formulation of lubricant oils and structure-property relationships. 